\title{
Measuring marginal risk contributions in credit portfolios
}

\author{
Paul Glasserman \\ 403 Uris Hall, Columbia Business School, New York, NY I0027, USA
}

\begin{abstract}
We consider the problem of decomposing the credit risk in a portfolio into a sum of risk contributions associated with individual obligors or transactions. For some standard measures of risk - including value-at-risk and expected shortfall - the total risk can be usefully decomposed into a sum of marginal risk contributions from individual obligors. Each marginal risk contribution is the conditional expected loss from that obligor, conditional on a large loss for the full portfolio. We develop methods for calculating or approximating these conditional expectations. Ordinary Monte Carlo estimation is impractical for this problem because the conditional expectations defining the marginal risk contributions are conditioned on rare events. We develop three techniques to address this difficulty. First, we develop importance sampling estimators specifically designed for conditioning on large losses. Next, we use the analysis underlying the importance sampling technique to develop a hybrid method that combines an approximation with Monte Carlo. Finally, we take this approach a step further and develop a rough but fast approximation that dispenses entirely with Monte Carlo. We develop these methods in the Gaussian copula framework and illustrate their performance in multifactor models.
\end{abstract}

\section{Introduction}

For purposes of internal risk management, the measurement of portfolio credit risk is often just a first step in a more extensive process of allocating capital to transactions with counterparties. This process requires decomposing the total credit risk in a portfolio into individual risk contributions. Each risk contribution assigns part of the total risk to a particular transaction or counterparty, and these risk contributions are then used to allocate capital.

For a bank following the internal-ratings-based approach of the Basel Committee's New Capital Accord (Basel Committee on Bank Supervision 2003), the credit risk in a portfolio is summarized through its value-at-risk (VAR), a quantile of the loss distribution. Similar considerations apply if risk (or capital adequacy) is measured using standard deviation, expected shortfall or several other candidate measures of risk. We focus on VAR and expected shortfall.

This work is supported, in part, by the Risk Measurement Research Program of the FDIC Center for Financial Research and by NSF grants DMI0300044 and DMS0410234. The author thanks Rosalind Bennett and Paul Kupiec of the FDIC for their comments on an early draft of this paper and Jingyi Li for assistance with numerical experiments. 
Many authors (including Denault (2001); Garman (1999); Gourieroux et al (2000); Kalkbrener (2003); Kurth and Tasche (2003); Litterman (1999)) have noted that several commonly used risk measures - including VAR and expected shortfall - can be usefully decomposed as a sum of sensitivities. In this decomposition, each risk contribution can be interpreted as a marginal impact on total portfolio risk. The key property underlying this decomposition is positive homogeneity of the risk measure, a property that states that the risk (or capital requirement) of a portfolio scales in proportion to the size of the portfolio.

The marginal risk contributions associated with both VAR and expected shortfall can be represented as conditional expectations of losses on subportfolios, conditioned on events in the tail of the loss distribution for the full portfolio. The rarity of these tail events presents an obstacle to practical calculation of these conditional expectations. Each contribution depends on the probability of a rare event (a default) conditional on an even rarer event (an extreme loss for the portfolio).

To address the practical difficulties of calculating marginal risk contributions, we develop efficient Monte Carlo methods and approximations. The Monte Carlo methods use importance sampling to address difficulties associated with simulating rare events. This part of the paper builds on the method developed in Glasserman and Li (2005) for estimating the tail of the loss distribution in credit portfolios. The techniques underlying the importance sampling method also lead to approximations that can be used together with Monte Carlo or as an alternative. Throughout this paper, we work in the Gaussian copula model of credit risk (Gupton et al 1997; Li 2000), widely used both for measuring portfolio credit risk and for pricing credit derivatives.

We review the Gaussian copula model in Section 2 and discuss the representation of marginal risk contributions as conditional expectations in Section 3. Section 4 analyzes the estimation of conditional expectations using ordinary Monte Carlo; we use this as a benchmark. Section 5 reviews importance sampling for large loss levels based on shifting the factor mean in the Gaussian copula and Section 6 extends this approach to conditional expectations. Section 7 uses related ideas to shrink the factor variance (as well as shifting the factor mean) in the importance sampling procedure. Section 8 develops an asymptotic approximation for marginal risk contributions and a hybrid method that combines Monte Carlo with an approximation. Section 9 takes the approximation a step further and dispenses with Monte Carlo.

\section{Portfolio credit risk in the Gaussian copula model}

A key element of any model of portfolio credit risk is a mechanism for capturing dependence among obligors. In this section, we describe the widely used Gaussian copula model for portfolio credit risk. Our description follows that in Glasserman (2004).

We focus on the distribution of losses from default over a fixed horizon. The ingredients of this distribution are as follows: $m$ is the number of obligors to which 
portfolio is exposed; $Y_{k}$ is the default indicator for the $k$ th obligor equal to 1 if the $k$ th obligor defaults or 0 otherwise; $p_{k}$ is the marginal probability that the $k$ th obligor defaults; $c_{k}$ is the loss given default for the $k$ th obligor; $X_{k}=c_{k} Y_{k}$ is the loss from the $k$ th obligor; $L=X_{1}+\cdots+X_{m}$ is the total loss from defaults.

We assume the marginal default probabilities $p_{k}$ are known, either from credit ratings or from the market prices of corporate bonds or credit default swaps. We take the $c_{k}$ to be constants for simplicity, although it would suffice to know the distribution of the $k$ th loss $X_{k}$. This would allow for random recovery.

The Gaussian copula model provides a mechanism for specifying dependence among the default indicators $Y_{1}, \ldots, Y_{m}$. Dependence is introduced through a multivariate normal vector $\left(\xi_{1}, \ldots, \xi_{m}\right)$ of latent variables. Each default indicator is represented as

$$
Y_{k}=\mathbf{1}\left\{\xi_{k}>x_{k}\right\}, \quad k=1, \ldots, m
$$

with $x_{k}$ chosen to match the marginal default probability $p_{k}$. The threshold $x_{k}$ is sometimes interpreted as a default boundary of the type arising in the foundational work of Merton (1974). Without loss of generality, we take each $\xi_{k}$ to have a standard normal distribution and set $x_{k}=\Phi^{-1}\left(1-p_{k}\right)$, where $\Phi$ is the cumulative normal distribution. Thus,

$$
P\left(Y_{k}=1\right)=P\left(\xi_{k}>\Phi^{-1}\left(1-p_{k}\right)\right)=1-\Phi\left(\Phi^{-1}\left(1-p_{k}\right)\right)=p_{k}
$$

The correlations among the $\xi_{k}$ determine the dependence among the $Y_{k}$. The underlying correlations are specified through a factor model of the form

$$
\xi_{k}=a_{k 1} Z_{1}+\cdots+a_{k d} Z_{d}+b_{k} \epsilon_{k}
$$

in which:

- $Z_{1}, \ldots, Z_{d}$ are independent systematic risk factors, each having an $N(0,1)$ (standard normal) distribution;

- $\epsilon_{k}$ is an idiosyncratic risk associated with the $k$ th obligor, also $N(0,1)$ distributed;

- $a_{k 1}, \ldots, a_{k d}$ are the factor loadings for the $k$ th obligor.

- $b_{k}=\sqrt{1-\left(a_{k 1}^{2}+\cdots+a_{k d}^{2}\right)}$ so that $\xi_{k}$ is $N(0,1)$.

The underlying factors $Z_{j}$ are sometimes derived from economic variables (eg, industry or regional risk factors).

Write $a_{k}$ for the row vector $\left(a_{k 1}, \ldots, a_{k d}\right)$ of factor loadings for the $k$ th obligor. The correlation between $\xi_{k}$ and $\xi_{\ell}, \ell \neq k$, is given by $a_{k} a_{\ell}^{\top}$. The conditional default probability for the $k$ th obligor given the factor loadings $Z=$ $\left(Z_{1}, \ldots, Z_{d}\right)^{\top}$ is

$$
p_{k}(Z)=P\left(Y_{k}=1 \mid Z\right)=P\left(\xi_{k}>x_{k} \mid Z\right)=\Phi\left(\frac{a_{k} Z+\Phi^{-1}\left(p_{k}\right)}{b_{k}}\right)
$$




\section{Risk measures and marginal risk contributions}

The distribution of credit losses in a portfolio (ie, the distribution of $L$ ) is typically summarized through a scalar measure of risk. Two of the most commonly used risk measures are VAR and expected shortfall. The VAR associated with probability $1-\alpha(\mathrm{eg}, \alpha=1 \%)$ is the quantile

$$
V A R_{\alpha}=\inf \{x: P(L \geq x) \leq \alpha\}
$$

The corresponding expected shortfall is

$$
E S_{\alpha}=\mathrm{E}\left[L \mid L \geq V A R_{\alpha}\right]
$$

Value-at-risk is in more widespread use, but expected shortfall is coherent (in the sense of Artzner et al (1999)) whereas VAR is not. In particular, VAR is not in general subadditive, which means that the sum of the VARs for two portfolios may be less than the VAR for the combined portfolio.

The calculation of a portfolio risk measure is often followed by a process of allocating the risk to elements of the portfolio based on their marginal contribution to the total risk. This type of decomposition is used for capital allocation and for measuring risk-adjusted performance.

Several authors have shown that the marginal risk contributions associated with VAR and expected shortfall can be represented as conditional expectations. In more detail, consider increasing the exposure to the $k$ th obligor by an amount $\epsilon$. The resulting portfolio loss random variable $L^{\epsilon}$ is related to the original loss $L$ by

$$
L^{\epsilon}=L+\epsilon X_{k}
$$

where $X_{k}$ is the loss random variable for the $k$ th obligor. Let $V A R_{\alpha}^{\epsilon}$ and $E S_{\alpha}^{\epsilon}$ respectively denote the VAR and expected shortfall for $L^{\epsilon}$. Then, under appropriate conditions (eg, Kalkbrener et al (2004); Kurth and Tasche (2003)) the marginal VAR contribution of the $k$ th obligor is

$$
\left.\frac{\partial V A R_{\alpha}^{\epsilon}}{\partial \epsilon}\right|_{\epsilon=0}=\mathrm{E}\left[X_{k} \mid L=V A R_{\alpha}\right]
$$

provided $P\left(L=V A R_{\alpha}\right)>0$. The marginal contribution to expected shortfall is

$$
\left.\frac{\partial E S_{\alpha}^{\epsilon}}{\partial \epsilon}\right|_{\epsilon=0}=\mathrm{E}\left[X_{k} \mid L \geq V A R_{\alpha}\right]
$$

Thus, in both cases, the marginal risk contributions are conditional expectations of the individual loss random variables, conditioned on rare values of the portfolio loss $L$.

We will not present (or verify) conditions under which (3) and (4) are valid. Instead, we simply note that the expressions on the right do indeed decompose the 
total risk. In the case of VAR,

$$
\sum_{k=1}^{m} \mathrm{E}\left[X_{k} \mid L=V A R_{\alpha}\right]=\mathrm{E}\left[\sum_{k=1}^{m} X_{k} \mid L=V A R_{\alpha}\right]=\mathrm{E}\left[L \mid L=V A R_{\alpha}\right]=V A R_{\alpha}
$$

and for expected shortfall,

$$
\sum_{k=1}^{m} \mathrm{E}\left[X_{k} \mid L \geq V A R_{\alpha}\right]=\mathrm{E}\left[\sum_{k=1}^{m} X_{k} \mid L \geq V A R_{\alpha}\right]=\mathrm{E}\left[L \mid L \geq V A R_{\alpha}\right]=E S_{\alpha}
$$

The calculation of these marginal risk contributions presents practical difficulties precisely because of the rarity of the conditioning events and because the calculation needs to be repeated for every obligor $k=1, \ldots, m$.

\section{Monte Carlo estimates of conditional expectations}

Estimation of the marginal risk contributions (3) and (4) by Monte Carlo can be thought of as a two-phase procedure in which one first estimates VAR (and possibly also expected shortfall) and then estimates the risk contributions using the estimated VAR from the first phase in place of the true VAR in the conditional expectations (3) and (4).

For the Gaussian copula model, the first-phase problem is addressed by the importance sampling (IS) procedure developed in Glasserman and Li (2005). That procedure involves shifting the mean of the common factors $Z$ and then increasing the conditional default probabilities given the outcome of $Z$. In the numerical examples reported in Glasserman and Li (2005), IS allows precise estimation of quantiles at probabilities as small as 0.0001 (and even smaller) from as few as 1,000 replications. (Other approaches to IS for credit risk are discussed in Kalkbrener et al (2004); Morokoff (2004).)

Here we focus on the second-phase problem of estimating, for a given loss level $x, \mathrm{E}\left[X_{k} \mid L=x\right]$ and $\mathrm{E}\left[X_{k} \mid L \geq x\right]$. We can treat the two cases together by considering

$$
v_{k}=\mathrm{E}\left[X_{k} \mid L \in \mathcal{A}\right]
$$

with $\mathcal{A}=\{x\}$ or $\mathcal{A}=[x, \infty)$. When we condition on $L \in \mathcal{A}$, we assume $P(L \in$ $\mathcal{A})>0$. If $P(L=x)=0$, we can replace the condition $L=x$ with $|L-x|<\epsilon$ for some $\epsilon>0$ large enough to have $P(|L-x|<\epsilon)>0$.

We first consider the estimation of $v_{k}, k=1, \ldots, m$, using ordinary Monte Carlo simulation. Each replication of an ordinary simulation proceeds along the following steps:

1. generate independent $N(0,1)$ factor levels $Z_{1}, \ldots, Z_{d}$ and set $Z=$ $\left(Z_{1}, \ldots, Z_{d}\right)^{\top}$

2. for each obligor $k=1, \ldots, m$, generate an independent $N(0,1)$ variable $\epsilon_{k}$ and set $\xi_{k}=a_{k 1} Z_{1}+\cdots+a_{k d} Z_{d}+b_{k} \epsilon_{k}$; 
3. for each obligor $k=1, \ldots, m$, generate the default indicator $Y_{k}=\mathbf{1}\left\{\xi_{k}>\right.$ $\left.\Phi^{-1}\left(1-p_{k}\right)\right\}$ and the loss given default $c_{k}$. Set $X_{k}=Y_{k} c_{k}$ and $L=X_{1}+$ $\cdots+X_{m}$.

These steps are repeated to generate multiple independent replications.

Let $\left(X_{1}^{(i)}, \ldots, X_{m}^{(i)}\right), i=1, \ldots, n$, be $n$ iid replications of the vector of individual losses $X_{k}$ and let

$$
L^{(i)}=X_{1}^{(i)}+\cdots+X_{m}^{(i)}
$$

denote the total portfolio loss on the $i$ th replication. To estimate the risk contributions $v_{k}, k=1, \ldots, m$, we use

$$
\hat{v}_{k}=\frac{\sum_{i=1}^{n} X_{k}^{(i)} \mathbf{1}\left\{L^{(i)} \in \mathcal{A}\right\}}{\sum_{i=1}^{n} \mathbf{1}\left\{L^{(i)} \in \mathcal{A}\right\}}
$$

We take the ratio to be zero whenever the denominator is zero.

Applying the strong law of large numbers to the numerator and denominator in (5), we find that, with probability of 1 ,

$$
\hat{v}_{k} \rightarrow v_{k}, \quad k=1, \ldots, m
$$

again assuming $P(L \in \mathcal{A})>0$. To compare the precision of this estimator with the alternatives that we propose, we need an appropriate measure of its variability. This is provided by a central limit theorem and the accompanying confidence intervals. We record these in the following result. As $\hat{v}_{k}$ is a ratio estimator, we cannot simply use a sample standard deviation to measure its precision; we instead apply the method in the following proposition.

Proposition 1 Suppose $P(L \in \mathcal{A})>0$ and let

$$
\hat{\sigma}_{k}^{2}=\frac{n \sum_{i=1}^{n}\left(X_{k}^{(i)}-\hat{v}_{k}\right)^{2} \mathbf{1}\left\{L^{(i)} \in \mathcal{A}\right\}}{\left(\sum_{i=1}^{n} \mathbf{1}\left\{L^{(i)} \in \mathcal{A}\right\}\right)^{2}}
$$

taking the ratio to be zero whenever the denominator is zero. Then the distribution of

$$
\frac{\hat{v}_{k}-v_{k}}{\hat{\sigma}_{k} / \sqrt{n}}
$$

converges to the standard normal and

$$
\hat{v}_{k} \pm z \delta / 2 \frac{\hat{\sigma}_{k}}{\sqrt{n}}
$$

is an asymptotically valid $1-\delta$ confidence interval for $v_{k}$, with $\Phi\left(z_{\delta / 2}\right)=1-$ $\delta / 2$. 
The limiting distribution in the proposition follows from a general result on the asymptotic normality of nonlinear functions of sample means (eg, see Serfling (1980, p. 122)) combined with the fact that (6) is a consistent estimator of the asymptotic variance of $\hat{v}_{k}$. In fact, this univariate central limit theorem extends in a straightforward way to a multivariate central limit theorem for the vector $\left(\hat{v}_{1}, \ldots, \hat{v}_{m}\right)$, which could be used to form simultaneous confidence intervals for multiple estimates.

\section{Importance sampling for large loss levels}

The main difficulty in using the standard Monte Carlo estimators $\hat{v}_{k}$ to calculate marginal risk contributions is that very few replications produce portfolio losses $L$ with $L=x$ or $L \geq x$. In IS, we try to increase the frequency with which rare events occur by changing the distribution from which we sample; we then weight each observation by a likelihood ratio to correct for the change in distribution.

Glasserman and Li (2005) develop an importance sampling technique for the estimation of $P(L \geq x)$ (which here appears as the denominator in the estimation of expected shortfall contributions). Their method applies a change of distribution to the vector of factors $Z$ and then changes the conditional default probabilities for a given $Z$.

The likelihood ratio that corrects for changing the distribution of $Z$ from $N(0, I)$ to $N(\mu, \Sigma)$, with $|\Sigma|>0$, is

$$
|\Sigma|^{1 / 2} \exp \left(\frac{1}{2}\left[(Z-\mu)^{\top} \Sigma^{-1}(Z-\mu)-Z^{\top} Z\right]\right)
$$

If we change only the mean of $Z$ (so $\Sigma=I$ ), this simplifies to

$$
\exp \left(-\mu^{\top} Z+\frac{1}{2} \mu^{\top} \mu\right)
$$

The effectiveness of IS depends critically on the choice of $\mu$ (and $\Sigma$ ), to which we will return.

\subsection{Twisting the conditional default probabilities}

Given $Z$, the conditional default probability of the $k$ th obligor is $p_{k}(Z)$ in (2). We embed these conditional probabilities in a parametric family, parameterized by a scalar $\theta$, by setting

$$
p_{k}(\theta, Z)=\frac{p_{k}(Z) \mathrm{e}^{\theta c_{k}}}{1+p_{k}(Z)\left(\mathrm{e}^{\theta c_{k}}-1\right)}
$$

These "exponentially twisted" probabilities monotonically increase in $\theta$ : at $\theta=0$, we recover the original probability $p_{k}(Z)$; taking $\theta>0$ increases the conditional default probability; and taking $\theta<0$ decreases the conditional default probability. There are many ways one might consider increasing or decreasing the conditional default probabilities, but this particular transformation has several important features that make it effective. 
A special feature of (7) is the resulting form of the likelihood ratio. The likelihood ratio that corrects for changing the conditional default probability $p_{k}(Z)$ to some other probability $q_{k}$ is

$$
\left(\frac{p_{k}(Z)}{q_{k}}\right)^{Y_{k}}\left(\frac{1-p_{k}(Z)}{1-q_{k}}\right)^{\left(1-Y_{k}\right)}
$$

In the particular case of $q_{k}=p_{k}(\theta, Z)$, this becomes

$$
\left(\frac{p_{k}(Z)}{p_{k}(\theta, Z)}\right)^{Y_{k}}\left(\frac{1-p_{k}(Z)}{1-p_{k}(\theta, Z)}\right)^{\left(1-Y_{k}\right)}=\mathrm{e}^{-\theta Y_{k} c_{k}}\left(1+p_{k}(Z)\left(\mathrm{e}^{\theta c_{k}}-1\right)\right)
$$

As the default indicators $Y_{1}, \ldots, Y_{m}$ are conditionally independent for a given $Z$, the (conditional) likelihood ratio for changing all the default probabilities is just the product of the individual (conditional) likelihood ratios, which can be written as

$$
\prod_{k=1}^{m} \mathrm{e}^{-\theta Y_{k} c_{k}}\left(1+p_{k}(Z)\left(\mathrm{e}^{\theta c_{k}}-1\right)\right)=\exp (-\theta L+\psi(\theta, Z))
$$

Here, we have used the fact that $L=Y_{1} c_{1}+\cdots+Y_{m} c_{m}$ and introduced the conditional cumulant generating function of $L$,

$$
\psi(\theta, z)=\log \mathrm{E}\left[\mathrm{e}^{\theta L} \mid Z=z\right]=\sum_{k=1}^{m} \log \left(1+p_{k}(Z)\left(\mathrm{e}^{\theta c_{k}}-1\right)\right)
$$

The special form of the likelihood ratio in (8) - depending on the default indicators $Y_{1}, \ldots, Y_{m}$ only through the total loss $L-$ is a consequence of the parametric specification in (7).

Glasserman and $\mathrm{Li}$ (2005) choose the parameter $\theta$ as a function of the factors $Z$ and the loss threshold $x$. Let $\theta_{x}(z)$ be the unique solution to the equation

$$
\frac{\partial}{\partial \theta} \psi(\theta, z)=x
$$

A unique solution indeed exists because, for all $z$, the derivative increases from $-\infty$ to $\infty$ as $\theta$ increases from $-\infty$ to $\infty$. It follows from (9) and (7) that the derivative on the left-hand side of this equation can be rewritten using the $p_{k}(\theta, z)$ in (7) as

$$
\frac{\partial}{\partial \theta} \psi(\theta, z)=\sum_{k=1}^{m} p_{k}(\theta, z) c_{k}
$$

Thus, setting $\theta=\theta_{x}(z)$ in (7) adjusts the conditional default probabilities just enough to make the conditional expected loss equal to $x$, in the sense that

$$
\sum_{k=1}^{m} p_{k}\left(\theta_{x}(z), z\right) c_{k}=x
$$


If the threshold $x$ is larger than the conditional expected loss $\mathrm{E}[L \mid Z=z]$, then $\theta_{x}(z)>0$ and the twisted conditional default probabilities $p_{k}\left(\theta_{x}(z), z\right)$ are greater than the original conditional default probabilities $p_{k}(z)$. If $x<\mathrm{E}[L \mid Z=z]$, then $\theta_{x}(z)<0$ and $p_{k}\left(\theta_{x}(z), z\right)<p_{k}(z)$. In estimating $P(L>x)$, Glasserman and Li (2005) use $\theta_{x}^{+}(z)=\max \left\{0, \theta_{x}(z)\right\}$, because in generating large values of $L$ there is no advantage to reducing the conditional default probabilities. However, negative values of $\theta_{x}(z)$ are useful in estimating conditional expectations given $L=x$.

\subsection{Shifting the factor mean}

Equation (7) specifies how we apply importance sampling to the default probabilities conditional on the outcome $Z$ of the factors. The method of Glasserman and $\mathrm{Li}$ (2005) also applies importance sampling to the factors themselves.

In estimating a tail probability $P(L>x)$, a particularly effective IS distribution for the factors $Z$ would be the probability density proportional to the function

$$
z \mapsto P(L>x \mid Z=z) \exp \left(-z^{\top} z / 2\right)
$$

If we formulate the calculation of $P(L>x)$ as a problem of integrating over the distribution of $Z$, then the first factor on the right is the integrand and the second factor is (up to a normalization constant) the multivariate normal density of $Z$. Sampling from this density is difficult, so we approximate it by a multivariate normal distribution $N(\mu, I)$. We would like to choose $\mu$ so that the mode of the multivariate normal coincides with the mode of (12). In other words, we would like to choose $\mu$ as the solution to

$$
\max _{z} P(L>x \mid Z=z) \exp \left(-z^{\top} z / 2\right)
$$

Even this approximation is not quite feasible because $P(L>x \mid Z=z)$ is difficult to evaluate, so we use a further approximation. Define

$$
F_{x}^{o}(z)=\max _{\theta}\{\psi(\theta, z)-x \theta\}=\psi\left(\theta_{x}(z), z\right)-x \theta_{x}(z)
$$

and

$$
F_{x}(z)=\max _{\theta \geq 0}\{\psi(\theta, z)-x \theta\}=\psi\left(\theta_{x}^{+}(z), z\right)-x \theta_{x}^{+}(z)
$$

These functions are illustrated for a homogeneous, single-factor model (with $p_{k} \equiv 0.02, c_{k} \equiv 1, a_{k 1} \equiv 0.3, m=100$ and $\left.x=10\right)$ in Figure 1. As shown in Glasserman and $\mathrm{Li}(2005), F_{x}$ provides an upper bound on the conditional tail of $L$ in the sense that

$$
P(L>x \mid Z=z) \leq \mathrm{e}^{F_{x}(z)}
$$

This upper bound also serves as a rough approximation. Using this approximation in (13) leads us to choose $\mu$ as the solution to

$$
\max _{z}\left\{F_{x}(z)-\frac{1}{2} z^{\top} z\right\}
$$


FIGURE I Graphs of the functions $F_{x}(z), F_{x}^{o}(z)$ and $F_{x}(z)-z^{2} / 2$ for a single-factor, homogeneous portfolio with $p_{k} \equiv 0.02, c_{k} \equiv 1, a_{k 1} \equiv 0.3, m=100$ and $x=10$. The point $\mu_{*}$ maximizes $F_{x}(z)-z^{2} / 2$, and $z_{*}$ is the smallest point at $F_{x}(z)=0$.

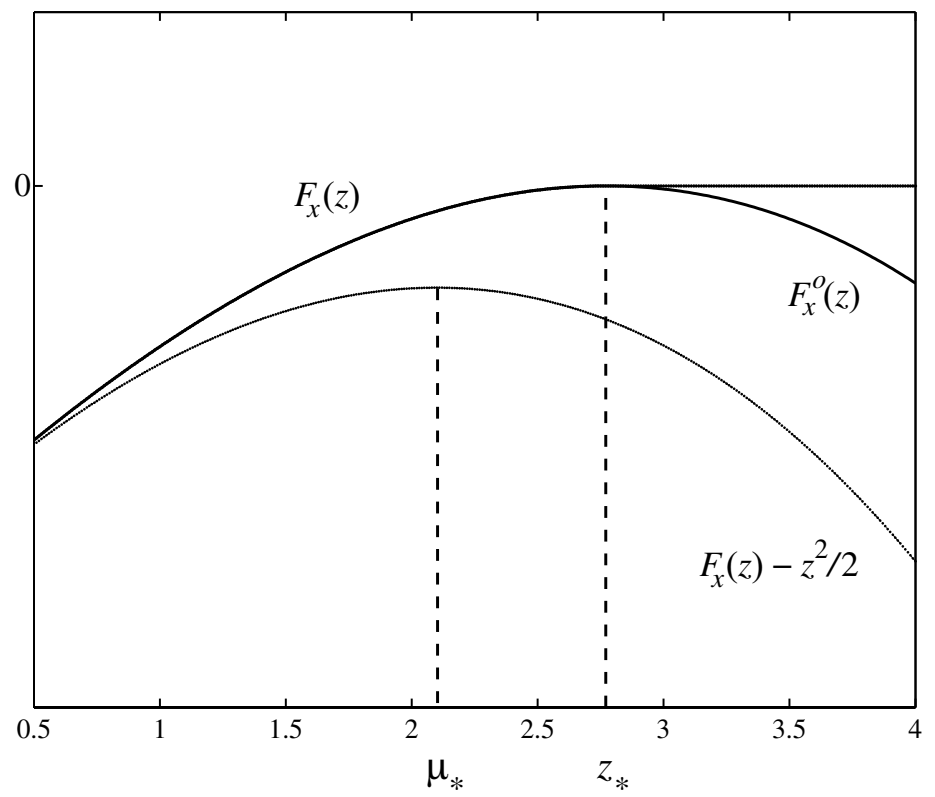

Glasserman and $\mathrm{Li}(2005)$ use the solution $\mu_{*}$ as the new mean of the factors in their IS procedure. There is just one maximizer in a single-factor model. In multifactor models, it is possible to have multiple solutions. For simplicity, we will assume a unique solution $\mu_{*}$ in our discussion.

A second point of interest for IS is the solution to

$$
\min _{z} z^{\top} z \quad \text { subject to } \quad F_{x}(z)=0
$$

In Figure 1, this is the smallest $z$ at which $F_{x}$ reaches zero and also the only point at which $F_{x}^{o}(z)=0$. This point is labeled $z_{*}$. As $F_{x}^{o}\left(z_{*}\right)=0$, (14) implies that $\theta_{x}\left(z_{*}\right)=0$, which means that $\mathrm{E}\left[L \mid Z=z_{*}\right]=x$. Thus, shifting the mean of $Z$ to $z_{*}$ is potentially attractive for IS. In fact, Glasserman and Li (2005) show that in a homogeneous, single-factor model, $\mu_{*} / z_{*} \rightarrow 1$ as the size of the portfolio increases. In an asymptotic sense, all candidate means between $\mu_{*}$ and $z_{*}$ are equally effective and, indeed, optimal. Glasserman et al (2005) prove asymptotic optimality of an importance sampling method for multifactor models based on a mixture of mean shifts that generalize $z_{*}$. 


\section{Importance sampling for conditional expectations}

We now return to the problem of estimating marginal risk contributions of the form $\mathrm{E}\left[X_{k} \mid L \in \mathcal{A}\right]$, with $\mathcal{A}=\{x\}$ or $\mathcal{A}=[x, \infty)$. In both cases, we assume $P(L \in$ $\mathcal{A})>0$ and use the representation

$$
r_{k} \equiv \mathrm{E}\left[X_{k} \mid L \in \mathcal{A}\right]=\frac{\mathrm{E}\left[X_{k} \mathbf{1}\{L \in \mathcal{A}\}\right]}{P(L \in \mathcal{A})}
$$

We apply importance sampling to estimate the numerator and the denominator. To keep the discussion generic, let $\tilde{P}$ and $\tilde{E}$ denote probability and expectation under a different probability measure with the same null sets as $P$. Write $\ell$ for the likelihood ratio $\mathrm{d} P / \mathrm{d} \tilde{P}$. Then

$$
r_{k}=\frac{\tilde{\mathrm{E}}\left[X_{k} \ell \mathbf{1}\{L \in \mathcal{A}\}\right]}{\tilde{\mathrm{E}}[\ell \mathbf{1}\{L \in \mathcal{A}\}]}
$$

Let $\left(X_{1}^{(i)}, \ldots, X_{m}^{(i)}\right), i=1, \ldots, n$, denote independent replications generated under $\tilde{P}$ and let $L^{(i)}=X_{1}^{(i)}+\cdots+X_{m}^{(i)}$. Let $\ell^{(i)}$ denote the likelihood ratio on the $i$ th replication. The estimator

$$
\hat{r}_{k}^{\mathrm{IS}}=\frac{\sum_{i=1}^{n} X_{k}^{(i)} \ell^{(i)} \mathbf{1}\left\{L^{(i)} \in \mathcal{A}\right\}}{\sum_{i=1}^{n} \ell^{(i)} \mathbf{1}\left\{L^{(i)} \in \mathcal{A}\right\}}
$$

converges to $r_{k}$ with a probability of 1 . We can assess the precision of this estimator using confidence intervals calculated in accordance with the following proposition.

Proposition 2 Suppose $P(L \in \mathcal{A})>0$ and

$$
\tilde{\mathrm{E}}\left[X_{k}^{2} \ell^{2} \mathbf{1}\{L \in \mathcal{A}\}\right]<\infty \text { and } \tilde{\mathrm{E}}\left[\ell^{2} \mathbf{1}\{L \in \mathcal{A}\}\right]<\infty
$$

Let

$$
\hat{\sigma}_{k}^{\mathrm{IS}}=\left(\frac{n \sum_{i=1}^{n}\left(X_{k}^{(i)} \ell^{(i)}-\hat{r}_{k}^{\mathrm{IS}} \ell^{(i)}\right)^{2} \mathbf{1}\left\{L^{(i)} \in \mathcal{A}\right\}}{\left(\sum_{i=1}^{n} \ell^{(i)} \mathbf{1}\left\{L^{(i)} \in \mathcal{A}\right\}\right)^{2}}\right)^{1 / 2}
$$

taking the ratio to be zero whenever the denominator is zero. Then with the $X_{k}^{(i)}$ sampled under $\tilde{P}$, the distribution of

$$
\frac{\hat{r}_{k}^{\mathrm{IS}}-r_{k}}{\hat{\sigma}_{k}^{\mathrm{IS}} / \sqrt{n}}
$$

converges to the standard normal and

$$
\hat{r}_{k}^{\mathrm{IS}} \pm z_{\delta / 2} \frac{\hat{\sigma}_{k}^{\mathrm{IS}}}{\sqrt{n}}
$$

is an asymptotically valid $1-\delta$ confidence interval for $r_{k}$. 


\subsection{Value-at-Risk contributions}

We now specialize to conditional expectations of the form $\mathrm{E}\left[X_{k} \mid L=x\right]$, as required for VAR contributions. We apply importance sampling to the estimation of both the numerator $\mathrm{E}\left[X_{k} \mathbf{1}\{L=x\}\right]$ and the denominator $P(L=x)$. We use the general class of IS procedures described in Section 5 in which we shift the mean of the factors $Z$ and then exponentially twist the conditional default probabilities, for a given $Z$. To complete the specification of the method, we need to select a new mean for $Z$ and a conditional twisting parameter $\theta$.

For the conditional twisting parameter we use $\theta_{x}(z)$ rather than its positive part

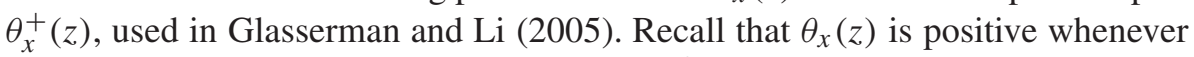
$\mathrm{E}[L \mid Z=z]$ is less than $x$. Thus, $\theta_{x}(z)$ and $\theta_{x}^{+}(z)$ differ only when $\mathrm{E}[L \mid Z=z]$ exceeds $x$. In estimating $P(L>x)$, large values of $L$ are useful, so there is no need to shrink the conditional expected loss back to $x$ if $\mathrm{E}[L \mid Z=z]>x$. However, in estimating $P(L=x)$ or expectations conditioned on $L=x$, we want to force the sampling procedure to generate more samples in which the loss $L$ exactly equals $x$. By using $\theta_{x}(z)$ rather than $\theta_{x}^{+}(z)$, we ensure that the expected loss, for a given $Z$, is equal to $x$ regardless of the outcome of $Z$. This is simply a restatement of (11).

For the new factor mean, we use $\mu_{*}$, the optimizer in (16). The resulting estimator of $\mathrm{E}\left[X_{k} \mathbf{1}\{L=x\}\right]$ is the sample mean of independent replications of

$$
\begin{aligned}
& X_{k} \ell \mathbf{1}\{L=x\} \\
& \quad=X_{k} \exp \left(-\theta_{x}(Z) L+\psi\left(\theta_{x}(Z), Z\right)\right) \exp \left(-\mu_{*}^{\top} Z-\mu_{*}^{\top} \mu_{*} / 2\right) \mathbf{1}\{L=x\}
\end{aligned}
$$

we estimate the denominator using independent replications of

$$
\ell \mathbf{1}\{L=x\}=\exp \left(-\theta_{x}(Z) L+\psi\left(\theta_{x}(Z), Z\right)\right) \exp \left(-\mu_{*}^{\top} Z-\mu_{*}^{\top} \mu_{*} / 2\right) \mathbf{1}\{L=x\}
$$

and, as in (18), the IS estimator of $\mathrm{E}\left[X_{k} \mid L=x\right]$ is the ratio of the averages of the two expressions over multiple replications. We detail the steps in Algorithm 1.

\subsection{Expected shortfall contributions}

We now modify the method of Section 6.1 to estimate $\mathrm{E}\left[X_{k} \mid L \geq x\right]$. The structure of the algorithm is the same, except that now we use $\theta_{x}^{+}(Z)$ rather than $\theta_{x}(Z)$ : if $\mathrm{E}[L \mid Z]<x$, we adjust the default probabilities to increase the conditional expected loss to $x$, but if $\mathrm{E}[L \mid Z] \geq x$, we do not change the default probabilities. This is shown in Algorithm 2.

\subsection{Numerical examples}

We now illustrate the performance of Algorithms 1 and 2 through examples. Each example is a portfolio of $m=100$ obligors; we have obtained nearly identical results with $m=1,000$, so the size of the portfolio does not appear to have much effect on the quality of the estimators. (Indeed, the asymptotic optimality 


\section{ALGORITHM I}

1. Find $\mu_{*}$ by solving (16).

2. Repeat for replications $i=1, \ldots, n$ :

(a) generate $Z$ from $N\left(\mu_{*}, I\right)$;

(b) calculate $p_{k}(Z)$ as in (2), $k=1, \ldots, m$;

(c) solve for $\theta_{x}(Z)$ in (11) and calculate $p_{k}\left(\theta_{x}(Z), Z\right), k=1, \ldots, m$;

(d) set $Y_{k}=1$ with probability $p_{k}\left(\theta_{x}(Z), Z\right)$ and $Y_{k}=0$ otherwise, $k=1, \ldots, m$;

(e) calculate total loss $L=Y_{1} c_{1}+\cdots+Y_{m} c_{m}$;

(f) calculate likelihood ratio;

$$
\ell=\exp \left(-\theta_{x}(Z) L+\psi\left(\theta_{x}(Z), Z\right)-\mu_{*}^{\top} Z+\mu_{*}^{\top} \mu_{*} / 2\right)
$$

(g) set Num $_{k}=$ Num $_{k}+Y_{k} c_{k} \ell \mathbf{1}\{L=x\}$ and $\operatorname{Den}_{k}=\operatorname{Den}_{k}+\ell \mathbf{1}\{L=x\}$.

3. Return $\hat{r}_{k}=$ Num $_{k} / \operatorname{Den}_{k}$.

\section{ALGORITHM 2}

1. Find $\mu_{*}$ by solving (16).

2. Repeat for replications $i=1, \ldots, n$ :

(a) generate $Z$ from $N\left(\mu_{*}, I\right)$;

(b) calculate $p_{k}(Z)$ as in $(2), k=1, \ldots, m$;

(c) solve for $\theta_{x}(Z)$ in (11) and calculate $p_{k}\left(\theta_{x}^{+}(Z), Z\right), k=1, \ldots, m$;

(d) set $Y_{k}=1$ with probability $p_{k}\left(\theta_{x}^{+}(Z), Z\right)$ and $Y_{k}=0$ otherwise, $k=1, \ldots, m$;

(e) calculate total loss $L=Y_{1} c_{1}+\cdots+Y_{m} c_{m}$;

(f) calculate likelihood ratio;

$$
\ell=\exp \left(-\theta_{x}^{+}(Z) L+\psi\left(\theta_{x}^{+}(Z), Z\right)-\mu_{*}^{\top} Z+\mu_{*}^{\top} \mu_{*} / 2\right)
$$

(g) set Num $_{k}=\mathrm{Num}_{k}+Y_{k} c_{k} \ell \mathbf{1}\{L \geq x\}$ and $\operatorname{Den}_{k}=\operatorname{Den}_{k}+\ell \mathbf{1}\{L \geq x\}$.

3. Return $\hat{r}_{k}=$ Num $_{k} /$ Den $_{k}$. 
results in Glasserman and $\mathrm{Li}$ (2005) assume $m \rightarrow \infty$, suggesting that the method is even more effective in large portfolios.) The effect of varying the default probabilities $p_{k}$ is similar to the effect of varying the exposures $c_{k}$, so we keep the default probabilities fixed at $1 \%$. Generally speaking, the benefit of the IS estimators is greater for rarer events; increasing or decreasing the overall level of the default probabilities has roughly the same effect as varying the loss level $x$ in the conditional expectations.

EXAMPLE 1 In our first example, the obligors are independent. The exposures are

$$
c_{k}= \begin{cases}1, & k=1, \ldots, 20 \\ 4, & k=21, \ldots, 40 \\ 9, & k=41, \ldots, 60 \\ 16, & k=61, \ldots, 80 \\ 25, & k=81, \ldots, 100\end{cases}
$$

This is a deliberately lumpy profile that will lead to significant variation in marginal risk contributions.

A loss level of $x=80$ corresponds roughly to a $99.9 \%$ VAR because $P(L \geq$ 80 ) is about $0.1 \%$. The probability that the loss exactly equals 80 is about $0.03 \%$, so in calculating VAR contributions (conditional expectations given $L=x$ ) we are indeed conditioning on a rare event.

Figure 2 compares the performance of the IS estimators and ordinary Monte Carlo for VAR contributions (top) and expected shortfall contributions (bottom). In each case, the horizontal axis lists the obligors from 1 to 100 and the vertical axis shows the estimated risk contribution for each obligor. The two solid lines show $95 \%$ confidence intervals for each obligor using IS; the two dotted lines show $95 \%$ confidence intervals using ordinary Monte Carlo. Both are calculated using 250,000 replications. This rather large number of replications is needed to get meaningful confidence intervals using ordinary Monte Carlo because we are conditioning on rare events; far fewer replications would suffice using IS. Indeed, the figures clearly show a substantial reduction in variability using IS. From the IS estimates, it is evident that the risk contributions are the same for consecutive blocks of obligors, although this is not at all clear from the ordinary Monte Carlo estimates.

Table 1 gives a numerical summary of the results illustrated in Figure 2. The columns labeled "VAR" and "ES" show point estimates of marginal contributions based on VAR and expected shortfall, respectively, computed with IS. The columns labeled "Plain" and "IS" show estimates of the variance using ordinary Monte Carlo and IS respectively. Thus, their ratio gives the variance reduction achieved by IS. Each entry in the table is an average over the 20 identical obligors in the corresponding row. We did not observe any outcomes with $L=80$ in which any of the first 20 obligors defaulted using either IS or plain Monte Carlo, which is why two of the variance entries in the table are blank. 
FIGURE 2 Comparison of IS (solid) and ordinary Monte Carlo (dotted) estimates in Example I for: (a) VAR contributions; (b) expected shortfall contributions.
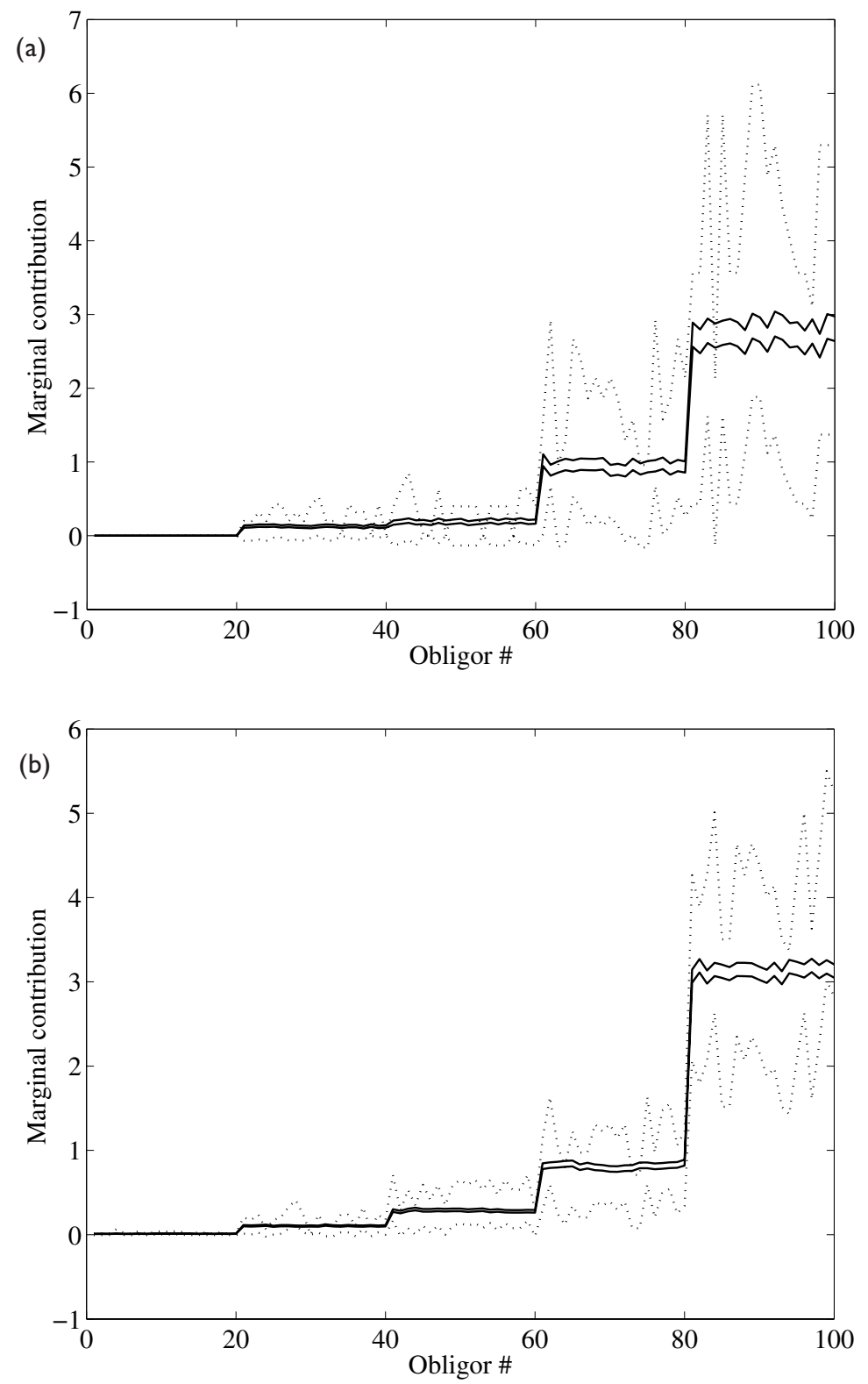
TABLE I Comparison of IS and plain Monte Carlo estimates for VAR contributions and expected shortfall contributions in Example I. The table shows point estimates and variance estimates, averaged over sets of identical obligors.

\begin{tabular}{clccccc}
\hline Obligor & VAR & Plain & IS & ES & Plain & IS \\
\hline $\mathrm{I}-20$ & 0 & & & $0.0 \mathrm{I}$ & $5 \times 10^{-5}$ & $2 \times 10^{-7}$ \\
$2 \mathrm{I}-40$ & 0.13 & $7 \times 10^{-3}$ & $\mathrm{I} \times 10^{-4}$ & 0.10 & $2 \times 10^{-3}$ & $1 \times 10^{-5}$ \\
$4 \mathrm{I}-60$ & 0.19 & $2 \times 10^{-2}$ & $2 \times 10^{-4}$ & 0.29 & $1 \times 10^{-2}$ & $6 \times 10^{-5}$ \\
$6 \mathrm{I}-80$ & 0.94 & $2 \times 10^{-1}$ & $2 \times 10^{-3}$ & $0.8 \mathrm{I}$ & $5 \times 10^{-2}$ & $3 \times 10^{-4}$ \\
$8 \mathrm{I}-100$ & 2.74 & $8 \times 10^{-1}$ & $7 \times 10^{-3}$ & 3.13 & $3 \times 10^{-1}$ & $2 \times 10^{-3}$ \\
\hline
\end{tabular}

EXAMPLE 2 We modify Example 1 by making it a single-factor model in which each obligor's latent variable $\xi_{k}$ has a correlation of 0.5 with the common factor. This dependence pushes more mass into the tail of the loss distributions, so we increase the loss threshold to $x=100$. The mean shift for the common factor is $\mu_{*}=2.00$. The results are illustrated in Figure 3. The average variance reduction ratio in this example, averaged over the 100 obligors, is about 20. Table 2 gives a more detailed comparison; see the discussion of Table 1 for an explanation of the entries in the table.

EXAMPLE 3 To test the effect of changing the exposures, we modify Example 2 by letting the $c_{k}$ increase linearly from 1 to 100 . We set the loss threshold at $x=$ 500 , which makes $P(L \geq x)$ about $1.1 \%$. The granularity of the $c_{k}$ makes $P(L=$ $x$ ) too small to be meaningful, so we replace the event $\{L=x\}$ with $\{|L-x| \leq 1\}$. This event has a probability of $0.02 \%$. The results are illustrated in Figure 4.

EXAMPLE 4 We now construct an 11-factor version of Example 2. The exposures are as in (20). The first factor is a market-wide factor and each obligor has a coefficient of 0.3 on this factor. In addition, the first 10 obligors have a coefficient of 0.8 on the second factor, the next 10 obligors have a coefficient of 0.8 on the next factor and so on. Thus, each obligor is sensitive to the market-wide factor and to one additional factor. The block structure of the dependence in this example ensures that the model cannot be well-approximated using a small number of factors. We set the loss threshold at $x=250$, for which $P(L \geq x)$ is about $0.1 \%$ (99.9\% VAR) and $P(L=x)$ is about $0.03 \%$.

The mean shift for this example is:

$$
\begin{aligned}
& \mu_{*}=(1.6214,0.0002,0.0002,0.0009,0.0009,0.0018 \text {, } \\
& 0.0018,0.0028,0.0028,2.1563,2.1563)^{\top}
\end{aligned}
$$

Observe that this primarily shifts the mean of the first factor and the last two. To interpret this solution, think of $\mu_{*}$ as the least costly outcome of $Z$ leading to large losses (losses near $x$ ) when the cost associated with $Z=z$ is $z^{\top} z / 2$. 
FIGURE 3 Comparison of IS (solid) and ordinary Monte Carlo (dotted) estimates in Example 2 for: (a) VAR contributions; (b) expected shortfall contributions.
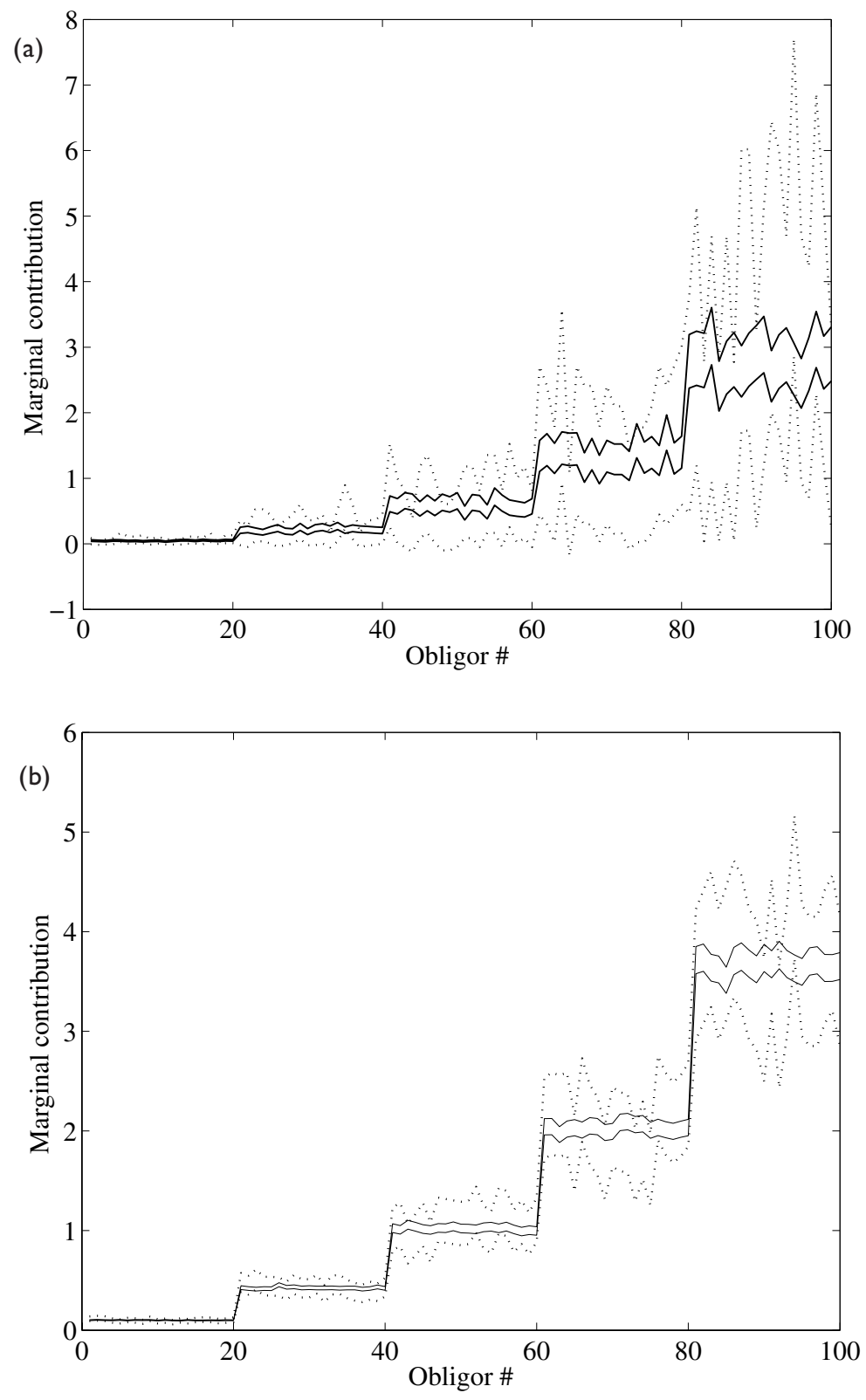
TABLE 2 Comparison of IS and plain Monte Carlo estimates for VAR contributions and expected shortfall contributions in Example 2. The table shows point estimates and variance estimates, averaged over sets of identical obligors.

\begin{tabular}{ccccccc}
\hline Obligor & VAR & Plain & IS & ES & Plain & IS \\
\hline $\mathrm{I}-20$ & 0.05 & $6 \times 10^{-4}$ & $3 \times 10^{-5}$ & 0.10 & $\mathrm{I} \times 10^{-4}$ & $5 \times 10^{-6}$ \\
$2 \mathrm{I}-40$ & 0.22 & $\mathrm{I} \times 10^{-2}$ & $6 \times 10^{-4}$ & 0.42 & $2 \times 10^{-3}$ & $9 \times 10^{-5}$ \\
$4 \mathrm{I}-60$ & 0.59 & $6 \times 10^{-2}$ & $3 \times 10^{-3}$ & 1.02 & $1 \times 10^{-2}$ & $5 \times 10^{-4}$ \\
$6 \mathrm{I}-80$ & 1.36 & $3 \times 10^{-1}$ & $1 \times 10^{-2}$ & 2.03 & $4 \times 10^{-2}$ & $2 \times 10^{-3}$ \\
$8 \mathrm{I}-100$ & 2.79 & $9 \times 10^{-1}$ & $4 \times 10^{-2}$ & 3.67 & $1 \times 10^{-1}$ & $5 \times 10^{-3}$ \\
\hline
\end{tabular}

A large outcome of the first factor is cost effective because the first factor affects all obligors. Large outcomes of the last two factors are cost effective not because those factor affect more obligors (each of the last 10 factors affects exactly 10 obligors), but rather because the last two factors affect the obligors with the highest exposures. Those are the obligors most likely to have defaulted when we condition on a large loss level. Also, observe that $\mu_{*}$ correctly detects the symmetry in the effect of consecutive pairs of factors. The results obtained using $\mu_{*}$ and 250,000 replications are illustrated Figure 5. The ordinary Monte Carlo results show not only wider confidence intervals but also a high degree of sampling variability in the estimated intervals. Table 3 reports point estimates and variance estimates; see the discussion of Table 1 for an explanation of the entries in the table.

\section{Shrinking the factor variance}

In the IS algorithms of the previous section, we shift the mean of the factors $Z$ but otherwise leave their distribution unchanged. The "optimal" density for $Z$ is that in (12); we chose the normal distribution to have the same mode as the optimal distribution. It is natural to consider whether changing the covariance matrix of the factors improves the approximation. Changing the covariance matrix is particularly appealing in estimating a conditional expectation given $L=x$ : to have more samples fall near a given point, we would like to reduce the variability of $Z$.

The optimal density (as in (12)) is the conditional density of $Z$, conditioned on either $L \geq x$ or $L=x$, depending on the context. One way to select an approximating normal distribution is to select a distribution that has the same first and second moments as the conditional density. These conditional moments are generally unknown but could be estimated from a preliminary set of runs. Using ordinary Monte Carlo, one could estimate these conditional moments by storing the values of $Z$ on which $L=x$ or $L \geq x$ and then calculating the sample mean and covariance of the stored factors. This would require a large number of replications. A more effective way would use importance sampling, starting with the distribution $N\left(\mu_{*}, I\right)$ used above. The conditional moments can be estimated 
FIGURE 4 Comparison of IS (solid) and ordinary Monte Carlo (dotted) estimates in Example 3 for: (a) VAR contributions; (b) expected shortfall contributions.
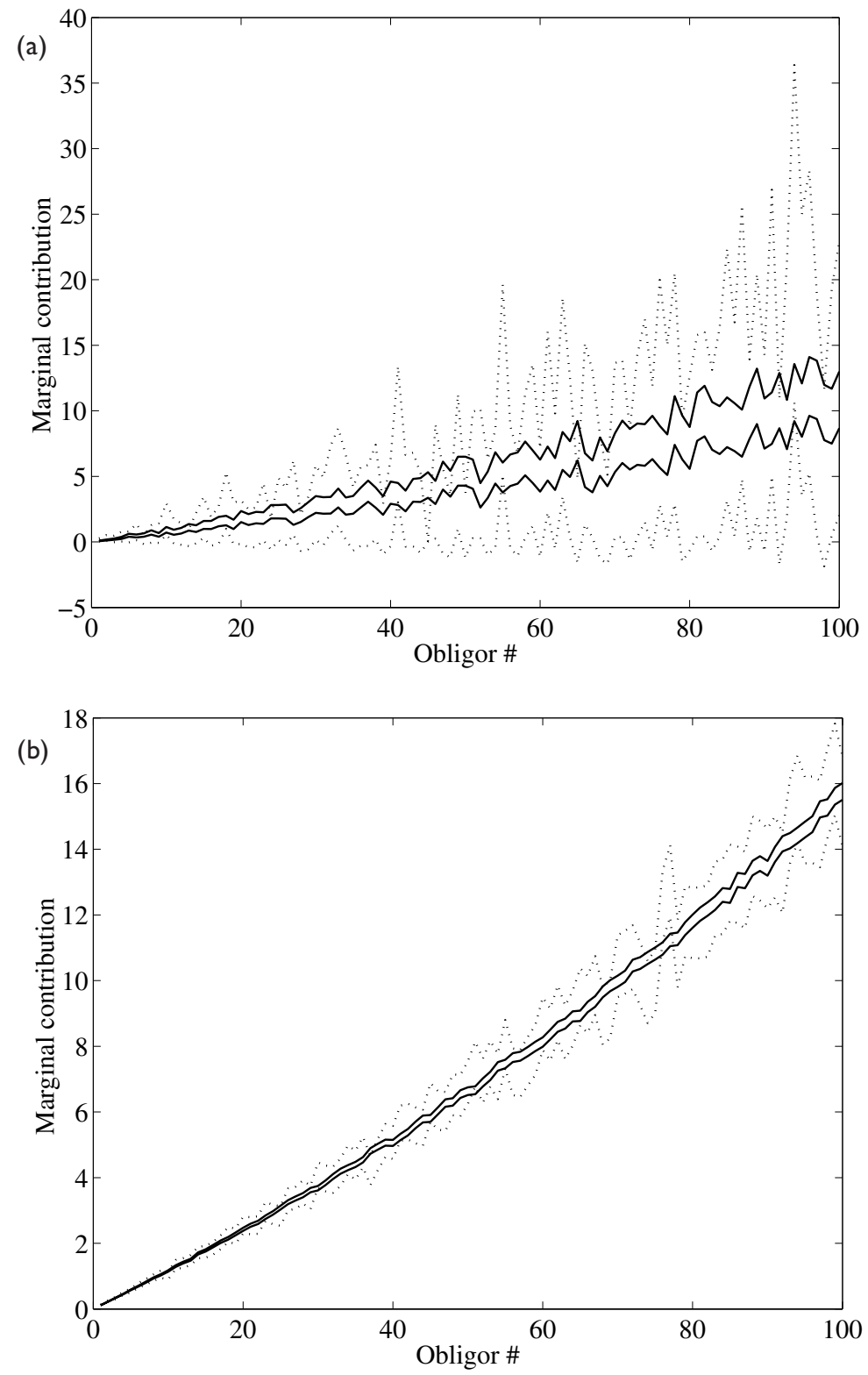
FIGURE 5 Comparison of IS (solid) and ordinary Monte Carlo (dotted) estimates in Example 4 for: (a) VAR contributions; (b) expected shortfall contributions.
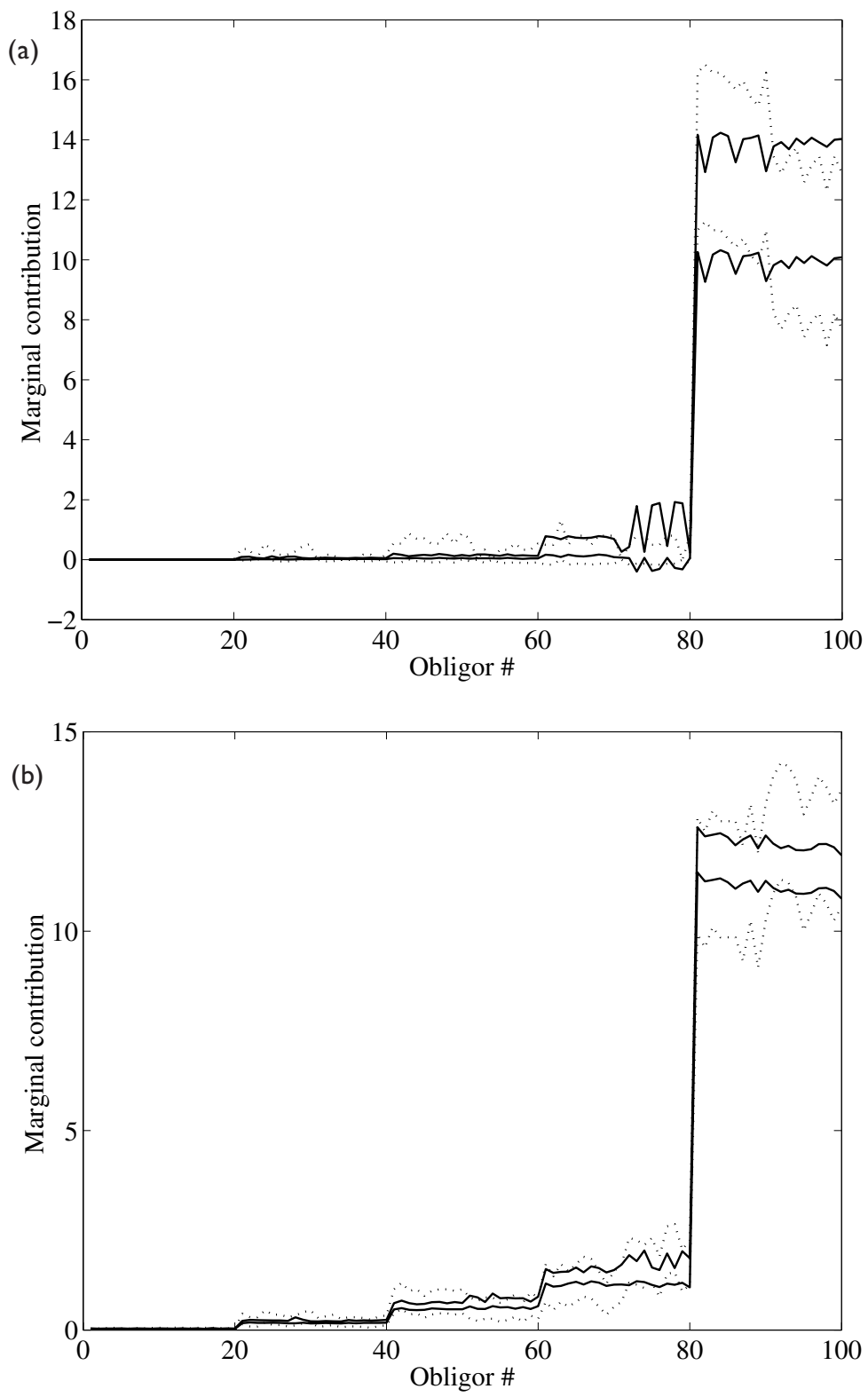
TABLE 3 Comparison of IS and plain Monte Carlo estimates for VAR contributions and expected shortfall contributions in Example 4. The table shows point estimates and variance estimates, averaged over sets of identical obligors.

\begin{tabular}{ccccccc}
\hline Obligor & VAR & Plain & IS & ES & Plain & IS \\
\hline $\mathrm{I}-20$ & 0.00 & & $2 \times 10^{-7}$ & 0.03 & $9 \times 10^{-5}$ & $8 \times 10^{-6}$ \\
$2 \mathrm{I}-40$ & 0.04 & $5 \times 10^{-3}$ & $2 \times 10^{-4}$ & $0.2 \mathrm{I}$ & $4 \times 10^{-3}$ & $3 \times 10^{-4}$ \\
$4 \mathrm{I}-60$ & 0.10 & $2 \times 10^{-2}$ & $7 \times 10^{-4}$ & 0.64 & $2 \times 10^{-2}$ & $3 \times 10^{-3}$ \\
$6 \mathrm{I}-80$ & 0.46 & $4 \times 10^{-2}$ & $9 \times 10^{-2}$ & 1.39 & $6 \times 10^{-2}$ & $2 \times 10^{-2}$ \\
$8 \mathrm{I}-100$ & $\mathrm{II} .90$ & 2 & $\mathrm{I}$ & 11.67 & $5 \times 10^{-1}$ & $8 \times 10^{-2}$ \\
\hline
\end{tabular}

using IS, producing estimates $\tilde{\mu}$ and $\tilde{\Sigma}$, say; these can then be used to update the IS distribution to $N(\tilde{\mu}, \tilde{\Sigma})$.

An alternative is to use the relation between the covariance matrix of a normal distribution and the Hessian (matrix of second derivatives) of the log density at its mode. The log of the $N(\mu, \Sigma)$ density (for non-singular $\Sigma$ ) is

$$
y \mapsto-\frac{1}{2}(y-\mu)^{\top} \Sigma^{-1}(y-\mu)+\text { constant }
$$

from which we see that the Hessian at $\mu$ is $-\Sigma^{-1}$.

In Figure 1, we select $\mu_{*}$ as the mode of $F_{x}^{o}(z)-z^{2} / 2$. This corresponds to approximating $\exp \left(F_{x}^{o}(z)-z^{2} / 2\right)$ by (a multiple of) the normal density $N\left(\mu_{*}, 1\right)$. In approximating this function by a normal density $N\left(\mu_{*}, \sigma^{2}\right)$, we can use the relation between the second derivative and the variance and choose $\sigma^{2}$ to satisfy

$$
-\frac{1}{\sigma^{2}}=\frac{\partial^{2}}{\partial z^{2}}\left(F_{x}^{o}(z)-\frac{1}{2} z^{2}\right)=\frac{\partial^{2}}{\partial z^{2}} F_{x}^{o}(z)-1
$$

In general, in the multi-factor case this corresponds to setting

$$
\Sigma=\left(I-\nabla_{2} F_{x}^{o}\left(\mu_{*}\right)\right)^{-1}
$$

assuming the inverse on the right exists and the resulting matrix is positive definite. The notation $\nabla_{2} F_{x}^{o}\left(\mu_{*}\right)$ indicates the Hessian of $F_{x}^{o}(\cdot)$ at $\mu_{*}$. Initial numerical experiments reported in $\mathrm{Li}$ (2004) indicate that this is an effective choice in estimating $P(L=x)$.

Appendix B of Glasserman (2004) shows that the Hessian of $F_{x}^{o}(\cdot)$ is given by

$$
\nabla_{2} F_{x}^{o}(z)=\nabla_{2} \psi\left(\theta_{x}, z\right)+\frac{\partial}{\partial \theta} \nabla \psi\left(\theta_{x}, z\right)^{\top} \nabla \theta_{x}(z)
$$

with

$$
\nabla \theta_{x}(z)=-\nabla \frac{\partial}{\partial \theta} \psi\left(\theta_{x}, z\right) / \frac{\partial^{2}}{\partial \theta^{2}} \psi\left(\theta_{x}, z\right)
$$

Thus, the Hessian can be evaluated purely in terms of derivatives of $\psi$. This is important because $\psi$ is given explicitly by (9), so its derivatives are easily evaluated. Evaluation of this part of (21) is thus practical. There is no guarantee 
FIGURE 6 IS confidence intervals for VAR contributions using shifted factor mean (dotted) and both shifted mean and modified factor variance (solid). Panel (a) shows Example 2 with $x=300$; panel (b) shows Example 3 and panel (c) shows Example 4.
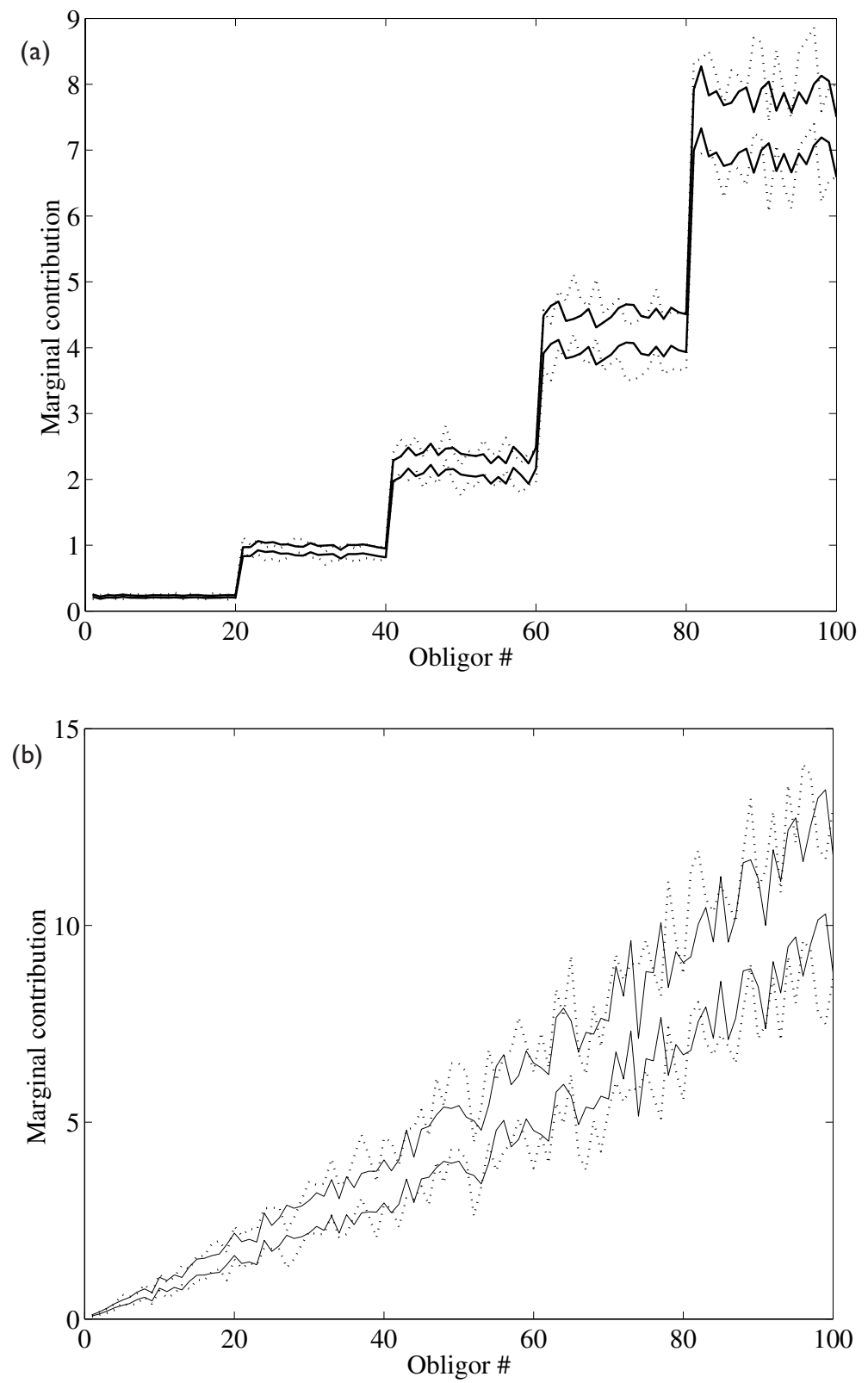
FIGURE 6 Continued.

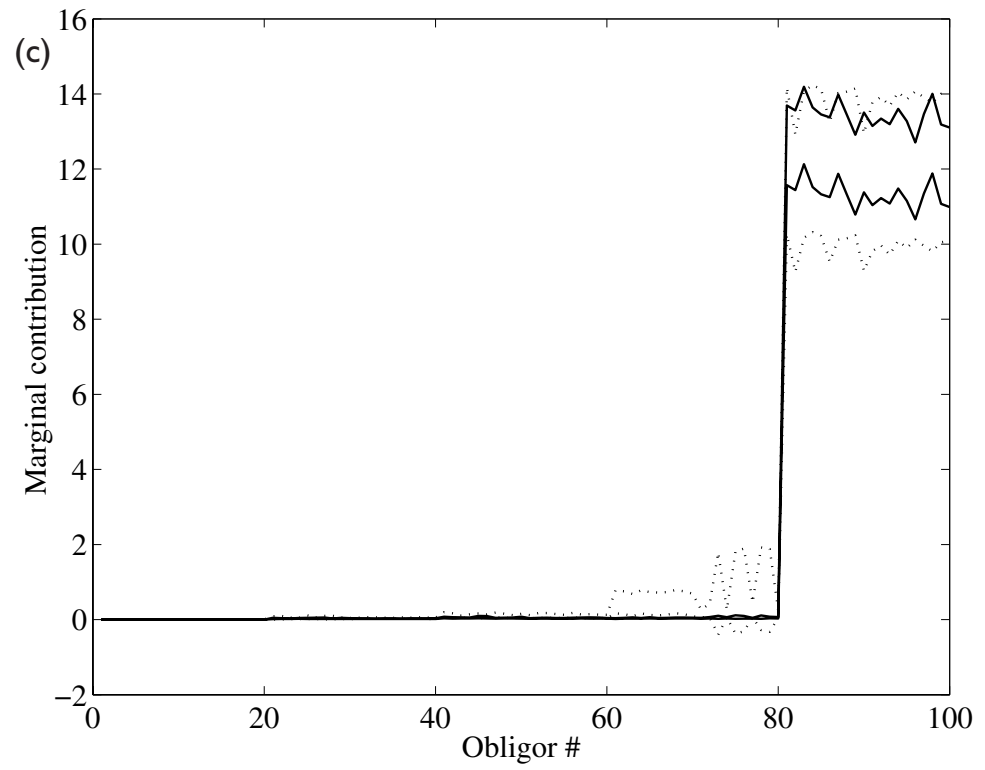

that the inverse in (21) exists; we have generally found that it does exist at sufficiently large values of $x$. Also, whether $\Sigma$ actually "shrinks" variance relative to the identity matrix depends on $\nabla_{2} F_{x}^{o}\left(\mu_{*}\right)$ being negative semidefinite, which requires that $F_{x}^{o}$ be concave near $\mu_{*}$. This property is also important in the approximations of Glasserman (2004).

The use of the modified variance in importance sampling is illustrated in Figure 6 for Examples 2-4, where we compare IS based solely on shifting the factor mean with IS based on shifting the mean and changing the covariance matrix. The figure indicates some modest further variance reduction from the change in covariance.

For Figure 6, we have moved the loss threshold to $x=300$ for Example 2, where the benefit of shrinking the factor standard deviation (from 1 to 0.294) should be greater. For Example 3, (21) gives a standard deviation of 0.3463. The covariance matrix we used for IS with Example 4 is

$\left(\begin{array}{rrrrrrrrrrr}0.791 & 0.000 & 0.000 & 0.003 & 0.003 & 0.006 & 0.006 & 0.009 & 0.009 & -0.296 & -0.296 \\ 0.000 & 1.002 & 0.000 & 0.000 & 0.000 & 0.000 & 0.000 & 0.000 & 0.000 & 0.000 & 0.000 \\ 0.000 & 0.000 & 1.002 & 0.000 & 0.000 & 0.000 & 0.000 & 0.000 & 0.000 & 0.000 & 0.000 \\ 0.003 & 0.000 & 0.000 & 1.009 & 0.000 & 0.000 & 0.000 & 0.000 & 0.000 & -0.001 & -0.001 \\ 0.003 & 0.000 & 0.000 & 0.000 & 1.009 & 0.000 & 0.000 & 0.000 & 0.000 & -0.001 & -0.001 \\ 0.006 & 0.000 & 0.000 & 0.000 & 0.000 & 1.020 & 0.000 & 0.000 & 0.000 & -0.003 & -0.003 \\ 0.006 & 0.000 & 0.000 & 0.000 & 0.000 & 0.000 & 1.020 & 0.000 & 0.000 & -0.003 & -0.003 \\ 0.009 & 0.000 & 0.000 & 0.000 & 0.000 & 0.000 & 0.000 & 1.031 & 0.000 & -0.004 & -0.004 \\ 0.009 & 0.000 & 0.000 & 0.000 & 0.000 & 0.000 & 0.000 & 0.000 & 1.031 & -0.004 & -0.004 \\ -0.296 & 0.000 & 0.000 & -0.001 & -0.001 & -0.003 & -0.003 & -0.004 & -0.004 & 1.048 & -0.823 \\ -0.296 & 0.000 & 0.000 & -0.001 & -0.001 & -0.003 & -0.003 & -0.004 & -0.004 & -0.823 & 1.048\end{array}\right)$


This covariance matrix differs from the identity matrix primarily in two ways: it assigns smaller variance to the first factor; and it introduces negative correlation between the factors whose means have been substantially increased - the first and the last two. In particular, the rather large negative covariance of -0.823 between the last two factors suggests that the event $\{L=x\}$ (in this case with $x=250$ ) occurs because of a large value of one of the last two factors, but not both. This phenomenon is also reflected in the pattern of estimated confidence intervals using ordinary Monte Carlo (the dotted lines) in Figure 5(a).

\section{Asymptotic approximation}

In this section, we develop a hybrid method that combines an asymptotic approximation with Monte Carlo. The asymptotic method approximates conditional risk contributions, given the factors; we use Monte Carlo solely to integrate the approximation over the distribution of the factors.

\subsection{A general approximation}

We formulate a rigorous result assuming homogeneous obligors and then apply the same techniques to develop approximations for general portfolios. In order to consider asymptotics as the portfolio size grows, we assume an infinite sequence $\left\{Z, X, X_{1}, X_{2}, \ldots\right\}$ with the $X_{i}$ interpreted (as before) as loss random variables, although not necessarily of the form $c_{i} Y_{i}$. We impose the following conditions:

(i) $X, X_{1}, X_{2}, \ldots$ are conditionally iid given $Z$;

(ii) $0 \leq X \leq b$ for some $b>0$, and $P(X<\epsilon \mid Z)$ and $P(X>b-\epsilon \mid Z)$ are positive with probability of 1 , for all $\epsilon>0$.

We define the conditional cumulant generating function of the $X_{i}$ by setting

$$
\psi_{X}(\theta, Z)=\log \mathrm{E}\left[\mathrm{e}^{\theta X} \mid Z\right], \quad-\infty<\theta<\infty
$$

The boundedness assumption in (ii) more than suffices to ensure finiteness of $\psi_{X}(\theta, Z)$. Let $G_{0}(\cdot, z)$ denote the conditional distribution of $X$ given $Z=z$,

$$
G_{0}(x, z)=P(X \leq x \mid Z=z) \text { for all } x
$$

We embed $G_{0}(\cdot, z)$ in an exponential family of distributions $\left\{G_{\theta}(\cdot, z),-\infty<\right.$ $\theta<\infty\}$ by setting

$$
G_{\theta}(x, z)=\int_{-\infty}^{x} \mathrm{e}^{\theta u-\psi_{X}(\theta, z)} \mathrm{d} G_{0}(u, z)
$$

These have the property (standard for exponential families) that

$$
\int_{-\infty}^{\infty} x \mathrm{~d} G_{\theta}(x, z)=\frac{\partial}{\partial \theta} \psi_{X}(\theta, z)
$$


For any $0<q<b$, let $\theta_{q}(z)$ be the unique solution to

$$
\frac{\partial}{\partial \theta} \psi_{X}\left(\theta_{q}(z), z\right)=q
$$

Assumption (ii) ensures that exactly one such solution indeed exists. The parameter $\theta_{q}(z)$ picks out the member of the exponential family $\left\{G_{\theta}(\cdot, z),-\infty<\theta<\right.$ $\infty$ \} whose mean is $q$.

Let $L_{m}=X_{1}+\cdots+X_{m}$. We let $m$ increase and consider the conditional distribution of any fixed set of losses $X_{1}, \ldots, X_{k}$, conditional on $Z$ and on either $L_{m}=q m$ or $L_{m} \geq q m$. A general class of results may be paraphrased as stating that the conditional distribution of, for example, $X_{1}$ converges to the exponentially twisted distribution with mean $q$. In other words, conditioning on the sum (in our case, the total loss) changes the distribution of the summands in a predictable way. We record precise versions of these assertions in the following proposition.

Proposition 3 Suppose (i) and (ii) hold and $0<q<b$.

(a) If $X$ has a lattice distribution given $Z=z$ with $P(X=q \mid Z=z)>0$, then for any fixed $k$, as $m \rightarrow \infty$,

$$
P\left(X_{1} \leq x_{1}, \ldots, X_{k} \leq x_{k} \mid L_{m}=q m, Z=z\right) \rightarrow \prod_{i=1}^{k} G_{\theta_{q}(z)}\left(x_{i}, z\right)
$$

Also, for $k \leq m$,

$$
\mathrm{E}\left[X_{k} \mid L_{m}=q m, Z=z\right]=\int_{-\infty}^{\infty} x \mathrm{~d} G_{\theta_{q}(z)}(x, z)=q
$$

(b) If, given $Z=z, X$ has a lattice distribution or a density, then

$$
P\left(X_{1} \leq x_{1}, \ldots, X_{k} \leq x_{k} \mid L_{m} \geq q m, Z=z\right) \rightarrow \prod_{i=1}^{k} G_{\theta_{q}^{+}(z)}\left(x_{i}, z\right)
$$

Also, as $m \rightarrow \infty$,

$$
\mathrm{E}\left[X_{k} \mid L_{m} \geq q m, Z=z\right] \rightarrow \int_{-\infty}^{\infty} x \mathrm{~d} G_{\theta_{q}^{+}(z)}(x, z)=q
$$

PROOF Once we condition on $Z=z$, the $X_{i}$ are conditionally iid and we can apply results for iid sequences. The limit in (23) then follows from Zabell (1980). The limit in (25) in the density case follows from Van Campenhout and Cover (1981) who also cite earlier work of Lanford. The lattice case is treated in Cover and Thomas (1991). The equality in (24) follows from the fact that $X_{1}, \ldots, X_{m}$ are exchangeable, given $L_{m}=q m$ and $Z=z$. The limit in (26) follows from (25) and the boundedness of the $X_{i}$ : convergence of distributions implies convergence of expectations for bounded random variables. 
If we interpret the limits in the proposition as approximations, then in each case the result states that in conditioning on a large loss for the whole portfolio (and also the factor outcome), we may approximate the conditional loss distribution for an individual obligor by exponentially twisting the original distribution loss distribution for that obligor.

\subsection{The hetereogeneous case}

The limits in Proposition 3 provide approximations for conditional expectations for fixed $m$. Although the proposition assumes homogeneous obligors, the approximations extend in a natural way to the heterogeneous case, as we now explain.

The limits in (25) and (26) suggest the approximation

$$
\mathrm{E}\left[X_{k} \mid L_{m} \geq q m, Z=z\right] \approx \mathrm{E}\left[X_{k} \exp \left(\theta_{q}^{+}(Z) L_{m}-m \psi_{X}\left(\theta_{q}^{+}(Z), Z\right)\right) \mid Z=z\right]
$$

In more detail, the right-hand side becomes

$$
\begin{aligned}
& \mathrm{E}\left[X_{k} \exp \left(\theta_{q}^{+}(Z) L_{m}-m \psi_{X}\left(\theta_{q}^{+}(Z), Z\right)\right) \mid Z=z\right] \\
& \quad=\mathrm{E}\left[X_{k} \exp \left(\theta_{q}^{+}(Z) X_{k}-\psi_{X}\left(\theta_{q}^{+}(Z), Z\right)\right) \mid Z=z\right] \\
& \quad=\int_{-\infty}^{\infty} x \mathrm{~d} G_{\theta_{q}^{+}(z)}(x, z)
\end{aligned}
$$

the first equality using the conditional independence of the $X_{i}$ and the second using the definition of $G_{\theta}$. From (28), we see that the approximation in (27) is the limit in (26).

For loss levels $X_{i}$ that are conditionally identically distributed, $m \psi_{X}$ is the conditional cumulant generating function of the total loss $L$; in generalizing (27) to the heterogeneous case, we therefore replace $m \psi_{X}$ with $\psi=\psi_{X_{1}}+\cdots+$ $\psi_{X_{m}}$, the conditional cumulant generating function of the total loss $L$. If we set the loss threshold $x$ equal to $q m$, then Equation (22) defining $\theta_{q}$ coincides with Equation (10) defining $\theta_{x}$. With these substitutions, we generalize (27) to

$$
\mathrm{E}\left[X_{k} \mid L \geq x, Z=z\right] \approx \mathrm{E}\left[X_{k} \exp \left(\theta_{x}^{+}(Z) L-\psi\left(\theta_{x}^{+}(Z), Z\right)\right) \mid Z=z\right]
$$

with $\theta_{x}$ as in (10). Written this way, the approximation does not rely on the $X_{i}$ being conditionally identically distributed.

The interpretation of (29) and of Proposition 3 is that conditioning on a large loss for the whole portfolio changes the distribution of the individual losses in a predictable way. In particular, conditional on a large loss $L \geq x$ (and on the factor outcome $Z=z$ ), the individual losses $X_{k}$ look like they came from the distribution determined by $\theta_{x}^{+}(z)$, rather than the original distribution.

Taking the expectation of both sides of (29), we get the approximation

$$
\mathrm{E}\left[X_{k} \mid L \geq x\right] \approx \mathrm{E}\left[X_{k} \exp \left(\theta_{x}^{+}(\tilde{Z}) L-\psi\left(\theta_{x}^{+}(\tilde{Z}), \tilde{Z}\right)\right)\right]
$$


Here, $\tilde{Z}$ has the distribution of $Z$ conditional on $L \geq x$. Comparing (23) and (25), we similarly obtain

$$
\mathrm{E}\left[X_{k} \mid L=x\right] \approx \mathrm{E}\left[X_{k} \exp \left(\theta_{x}(\tilde{Z}) L-\psi\left(\theta_{x}(\tilde{Z}), \tilde{Z}\right)\right)\right]
$$

but with $\tilde{Z}$ having the distribution of $Z$ conditional on $L=x$. The two approximations nearly coincide when $x$ is large (ie, when $\theta_{x}(z)>0$ ) because conditional on $L \geq x$, the total loss $L$ is likely to be very near $x$ if $x$ is large. In applying these ideas, we will introduce an adjustment that differentiates the two cases.

\subsection{A hybrid method}

We now specialize to the case $X_{k}=Y_{k} c_{k}$ in which $Y_{k}$ is a default indicator and $c_{k}$ is a fixed loss given default. In this setting, (31) becomes

$$
\mathrm{E}\left[X_{k} \mid L=x\right]=c_{k} \mathrm{E}\left[Y_{k} \mid L=x\right] \approx c_{k} \mathrm{E}\left[p_{k}\left(\theta_{x}(Z), Z\right)\right]
$$

with $p_{k}(\theta, z)$ as in (2). This suggests a hybrid method in which we use Monte Carlo to "integrate out" the factors $Z$, but conditional on $Z$ we use the approximation $p_{k}\left(\theta_{x}(Z), Z\right)$, rather than a ratio estimator as in (5). This has the enormous advantage in that it allows us to estimate a conditional expectation given $L=x$ from all replications, not just the few on which $L$ equals $x$.

To implement this, we need to generate $Z$ from the correct distribution. In passing from (29) to (30), we are integrating over the distribution of $Z$ given $L \geq x$ (or, in the case of (31), the distribution of $Z$ given $L=x$ ). So, in calculating the expectation on the right-hand side of (32), we need to generate $Z$ from the appropriate conditional distribution.

Let $\phi(z)$ denote the unconditional density of $Z$ and $\phi(z \mid L \geq x)$ the conditional density given $L \geq x$. The conditional density is given by Bayes' rule:

$$
\phi(z \mid L \geq x) \propto P(L \geq x \mid Z=z) \phi(z)
$$

In the case of multivariate normal $Z$, this becomes

$$
\phi(z \mid L \geq x) \propto P(L \geq x \mid Z=z) \exp \left(-z^{\top} z\right)
$$

Much as in our discussion of importance sampling, we approximate this distribution by a multivariate normal $N(\mu, I)$ with $\mu$ chosen to match the mode of the conditional distribution - more precisely, we choose $\mu$ as in (16). This leads to estimate $\mathrm{E}\left[Y_{k} c_{k} \mid L=x\right]$, combining the approximation $p_{k}\left(\theta_{x}(Z), Z\right)$ with Monte Carlo using the approximate conditional distribution of $Z$ as shown in Algorithm 3.

To approximate $\mathrm{E}\left[Y_{k} c_{k} \mid L \geq x\right]$, we make two modifications. The first is to replace $\theta_{x}(Z)$ with $\theta_{x}^{+}(Z)$ in Algorithm 3, Step 2(c). Recall that $\theta_{x}(z)$ is negative when the conditional expected loss $\mathrm{E}[L \mid Z=z]$ exceeds $x$. Using a negative value of the parameter makes sense in estimating a conditional expectation given $L=x$, 
ALGORITHM 3 Approximate VAR contributions.

1. Find $\mu_{*}$ by solving (16).

2. Repeat for replications $i=1, \ldots, n$ :

(a) generate $Z$ from $N\left(\mu_{*}, I\right)$;

(b) calculate $p_{k}(Z)$ as in (2), $k=1, \ldots, m$;

(c) solve for $\theta_{x}(Z)$ in (11) and calculate $p_{k}\left(\theta_{x}(Z), Z\right), k=1, \ldots, m$;

(d) set $\hat{v}_{k}=\hat{v}_{k}+p_{k}\left(\theta_{x}(Z), Z\right) c_{k}$.

3. Return $\hat{v}_{k}=\hat{v}_{k} / n$.

because doing so pulls large values of $L$ down towards $x$. However, for a conditional expectation given $L \geq x$, large values of $L$ are as useful as samples that land on $x$, so we replace $\theta_{x}(z)$ with zero when it is negative.

The second modification is a scaling adjustment at the last step. The asymptotics in Proposition 3 do not distinguish between conditioning on $L=x$ and $L \geq x$. In fact, the conditional default probabilities should be larger, on average, in the second case, so we correct for this.

Our correction is based on the following observations. If the approximation

$$
p_{k}\left(\theta_{x}^{+}(z), z\right) \approx P\left(Y_{k}=1 \mid Z=z, L \geq x\right)
$$

held exactly, then we would have equality between

$$
\mathrm{E}\left[\sum_{k=1}^{m} p_{k}\left(\theta_{x}^{+}(\tilde{Z}), \tilde{Z}\right) c_{k}\right] \text { and } \mathrm{E}[L \mid L \geq x]
$$

Let $\bar{M}$ denote the average value of $\sum_{k=1}^{m} p_{k}\left(\theta_{x}^{+}(Z), Z\right) c_{k}$ over $n$ replications, with $Z \sim N\left(\mu_{*}, I\right)$ or $Z \sim N\left(\mu_{*}, \Sigma\right)$ to approximate $\tilde{Z}$, which has the distribution of $Z$ conditional on $L \geq x$. We would like to make the adjustment

$$
p_{k}\left(\theta_{x}^{+}(Z), Z\right) \rightarrow p_{k}\left(\theta_{x}^{+}(Z), Z\right) \frac{\mathrm{E}[L \mid L \geq x]}{\bar{M}}
$$

to the approximate conditional default probabilities. We do not know $\mathrm{E}[L \mid L \geq x]$, but we can estimate it from the simulation itself. This is what the scaling factor does in Algorithm 4.

REMARKS (1) The scaling adjustment scales the estimated risk contributions of all obligors by the same amount, but this should be viewed as an approximation.

(2) Rather than solve for $\theta_{x}(z)$ and then take the positive part, one can first check if

$$
\sum_{k=1}^{m} p_{k}(z) c_{k}>x
$$


ALGORITHM 4 Approximate shortfall contributions.

1. Find $\mu_{*}$ by solving (16).

2. Repeat for replications $i=1, \ldots, n$.

(a) Generate $Z$ from $N\left(\mu_{*}, I\right)$.

(b) Calculate $p_{k}(Z)$ as in (2), $k=1, \ldots, m$.

(c) Solve for $\theta_{x}(Z)$ in (11) and calculate $p_{k}\left(\theta_{x}^{+}(Z), Z\right), k=1, \ldots, m$.

(d) Calculate scaling factor:

(i) set $M=M+\sum_{k=1}^{m} p_{k}\left(\theta_{x}^{+}(Z), Z\right) c_{k}$;

(ii) generate default indicators $Y_{1}, \ldots, Y_{m}$ using probabilities $p_{k}\left(\theta_{x}^{+}(Z), Z\right), k=1, \ldots, m$;

(iii) calculate loss $L$ and likelihood ratio $\ell$ and set $N=N+L \mathbf{1}\{L \geq$ $x\} \ell$ and $D=D+\mathbf{1}\{L \geq x\} \ell$.

(e) Set $\hat{s}_{k}=\hat{s}_{k}+p_{k}\left(\theta_{x}(Z), Z\right) c_{k}$.

3. Return $\tilde{s}_{k}=\hat{s}_{k} N /(D \times M)$.

and solve for $\theta_{x}(z)$ only if this holds. If this inequality fails, $\theta_{x}(z)$ is in $(-\infty, 0]$ and $\theta_{x}^{+}(z)=0$.

(3) In the algorithms above, the $\hat{v}_{k}$ are sample means of iid replications, so supplementing them with confidence intervals is straightforward using their sample standard deviations. The estimator of Algorithm 4 is slightly more complicated because of the multiplicative adjustment, but a central limit theorem for it can be established along the lines of Propositions 1 and 2.

As this method involves an approximation, its limiting normal distribution is centered at a point offset from the true risk contribution by an unknown bias. We therefore focus on the limiting variance, which can be used to form an asymptotically valid confidence interval for the limiting mean. Set $\bar{M}=M / n$, $\bar{N}=N / n$ and $\bar{D}=D / n$ with $M, N$ and $D$ as in the algorithm and let $\bar{s}_{k}=\hat{s}_{k} / n$, with $\hat{s}_{k}$ as in Step 2(e). Let $B_{k}$ be $n$ times the sample covariance matrix of the vector $\left(\bar{s}_{k}, \bar{N}, \bar{D}, \bar{M}\right)$, based on $n$ replications, and let

$$
G_{k, n}=\left(\frac{\bar{N}}{\bar{D} \bar{M}}, \frac{\bar{s}_{k}}{\bar{D} \bar{M}},-\frac{\bar{s}_{k} \bar{N}}{\bar{D}^{2} \bar{M}},-\frac{\bar{s}_{k} \bar{N}}{\bar{D} \bar{M}^{2}}\right)
$$

Then $G_{k, n} B_{k} G_{k, n}^{\top}$ is a consistent estimator of the asymptotic variance of $\tilde{s}_{k}$. This follows from a general result on the asymptotic normality of nonlinear functions of sample means (eg, as in Serfling (1980, p. 122)).

\subsection{Numerical examples}

We illustrate the performance of the asymptotic approximation using the example portfolios of Section 6.3. Figure 7 shows results for Example 1 (which has 
FIGURE 7 The dotted line shows the hybrid approximation. IS confidence intervals (solid lines) are included for reference. The graphs show: (a) VAR contributions; and (b) expected shortfall contributions in Example I.
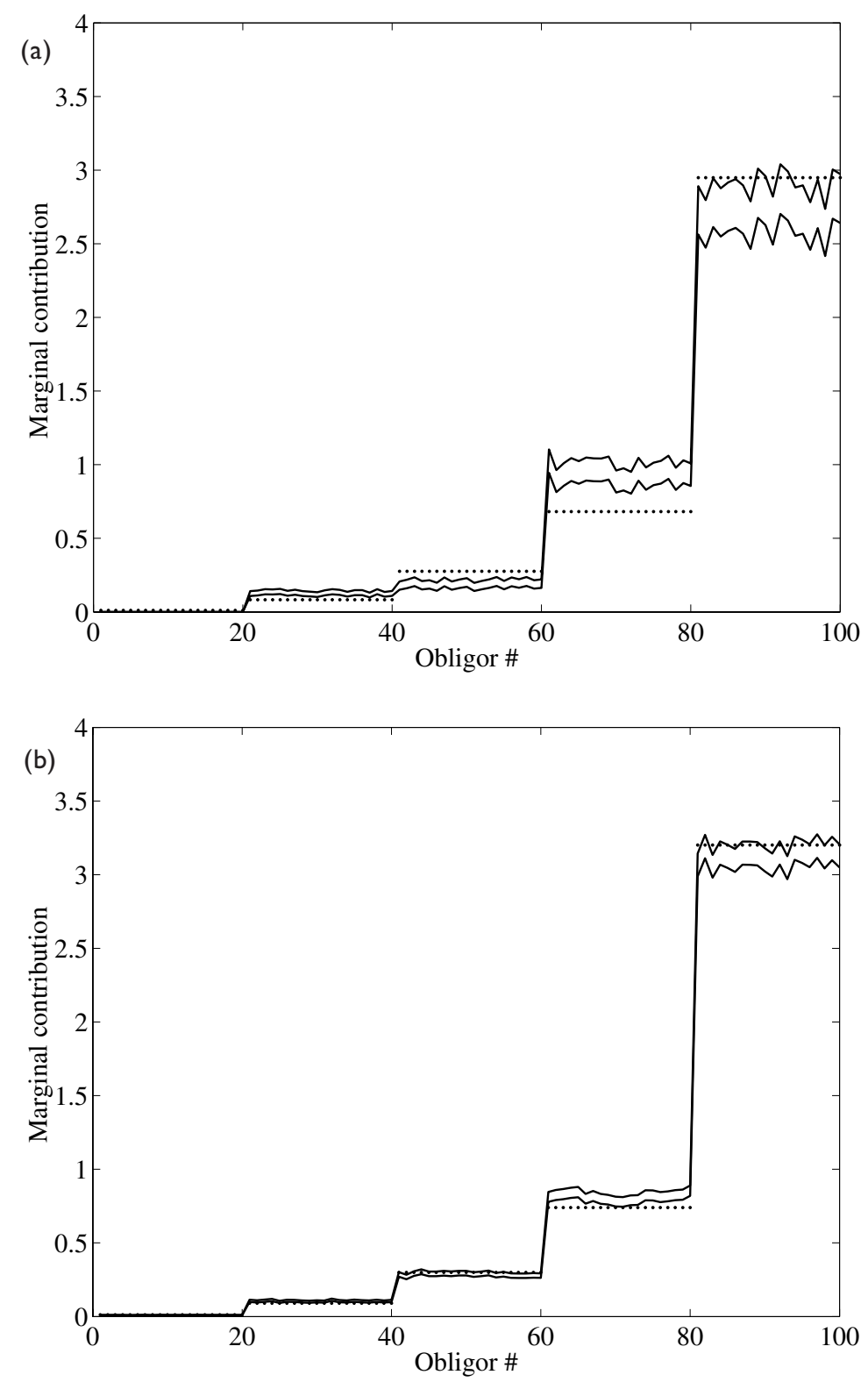
independent obligors), Figure 8 applies to the single-factor model in Example 2, Figure 9 shows results for Example 3 and results for the 11-factor model of Example 4 appear in Figure 10. Each figure compares the approximation with a $95 \%$ confidence interval estimated using importance sample. In each case, the approximation is sufficiently accurate to be useful.

The figures suggest that the asymptotic approximation is more accurate for VAR contributions (conditioned on $L=x$ ) than for expected shortfall contributions (conditioned on $L \geq x$ ). This makes sense, because the asymptotics in Proposition 3 implicitly approximate conditioning on $L \geq x$ by conditioning on $L=x$. The approximations in the figure distinguish between the two primarily by a scale factor: the final step in Algorithm 4 scales the risk contributions so they sum (approximately) to $\mathrm{E}[L \mid L \geq x]$.

An alternative way to approximate shortfall contributions $\mathrm{E}\left[X_{k} \mid L \geq x\right]$ would be to take a weighted average of approximate VAR contributions $\mathrm{E}\left[X_{k} \mid L=x_{i}\right]$ at several thresholds $x_{i}$. The weight for $\mathrm{E}\left[X_{k} \mid L=x_{i}\right]$ should approximate the probability that $L$ is closest to that $x_{i}$ given that $L \geq x$. These probabilities can be estimated using IS.

\section{Approximation without Monte Carlo}

The methods of Section 8 approximate the conditional default probability of each obligor (given a large loss level and the factor outcome), but use Monte Carlo to integrate out the distribution of the factors. In this section, we take the approximations one step further and dispense entirely with Monte Carlo. Rather than integrate over the distribution of factors, we evaluate the approximations of Section 8 at a single outcome of the factors - namely, the "most likely" outcome $\mu_{*}$ leading to large losses. Related ideas are investigated in Glasserman (2004) as a means of approximating the overall risk in a portfolio (as opposed to the individual risk contributions). As explained there, this may be viewed as a simplified application of the classical Laplace approximation for integrals. The full Laplace approximation could be applied using the candidate covariance matrix in (21), provided this matrix is indeed positive definite.

By evaluating the steps in Algorithm 3 at just the single outcome $Z=\mu_{*}$, we arrive at the approximation shown in Algorithm 5.

In Algorithm 4, one of the ways we distinguished between VAR contributions and expected shortfall contributions involved scaling the approximate contributions to set their sum approximately equal to $\mathrm{E}[L \mid L \geq x]$, or rather an estimate of this ratio. This is the scaling adjustment in Algorithm 4, calculated from the simulated losses themselves. Once we dispense entirely with Monte Carlo, we have no obvious mechanism for applying a similar adjustment. In the algorithm that follows, we adopt as a crude approximation

$$
\mathrm{E}[L \mid L \geq x] \approx x+\mathrm{E}[L]=x+\sum_{k=1}^{m} p_{k} c_{k}
$$


FIGURE 8 The dotted line shows the hybrid approximation. IS confidence intervals (solid lines) are included for reference. The graphs show: (a) VAR contributions; and (b) expected shortfall contributions in Example 2.
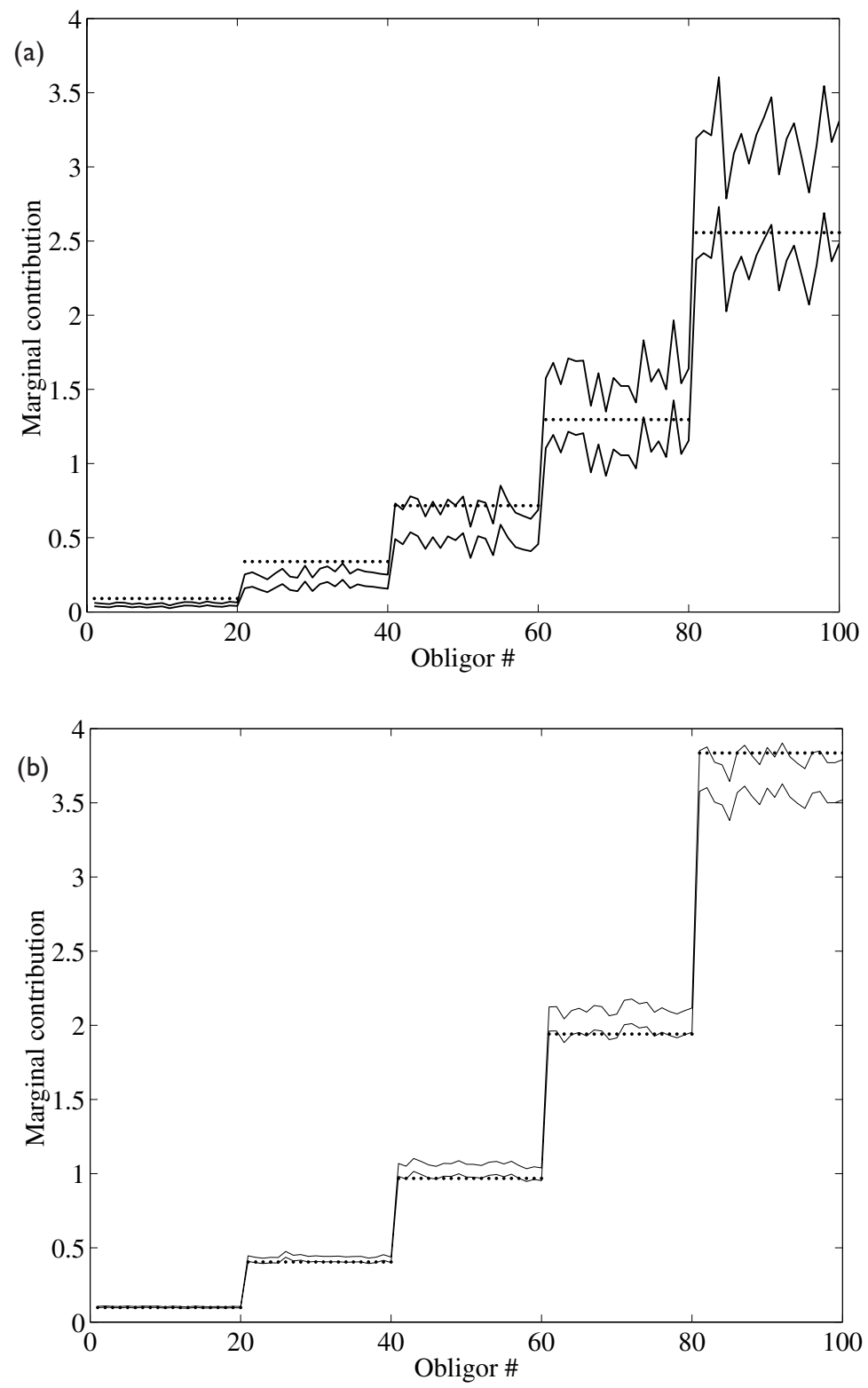
FIGURE 9 The dotted line shows the hybrid approximation. IS confidence intervals (solid lines) are included for reference. The graphs show: (a) VAR contributions; and (b) expected shortfall contributions in Example 2.
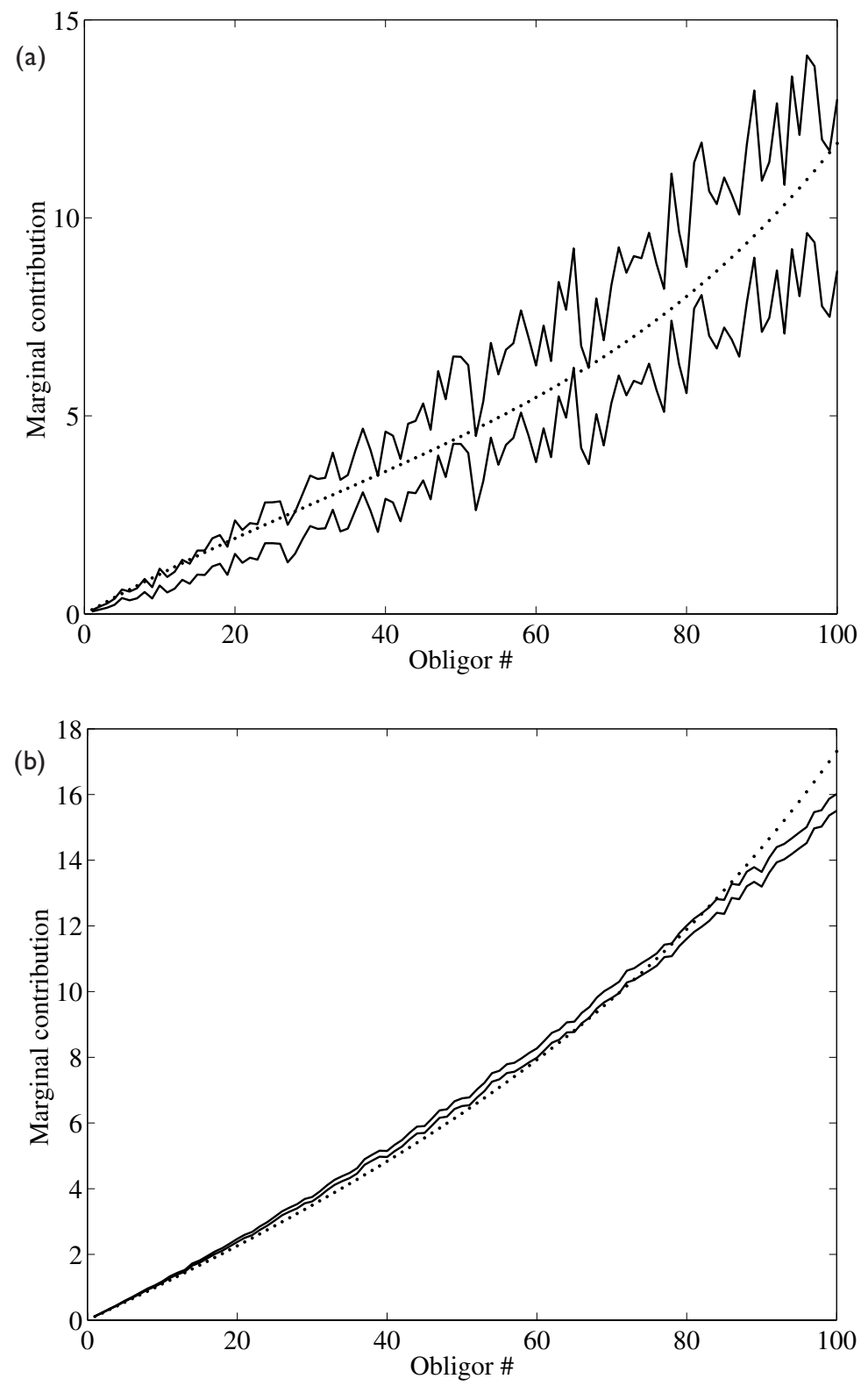
FIGURE 10 The dotted line shows the hybrid approximation. IS confidence intervals (solid lines) are included for reference. The graphs show: (a) VAR contributions; and (b) expected shortfall contributions in Example 4.
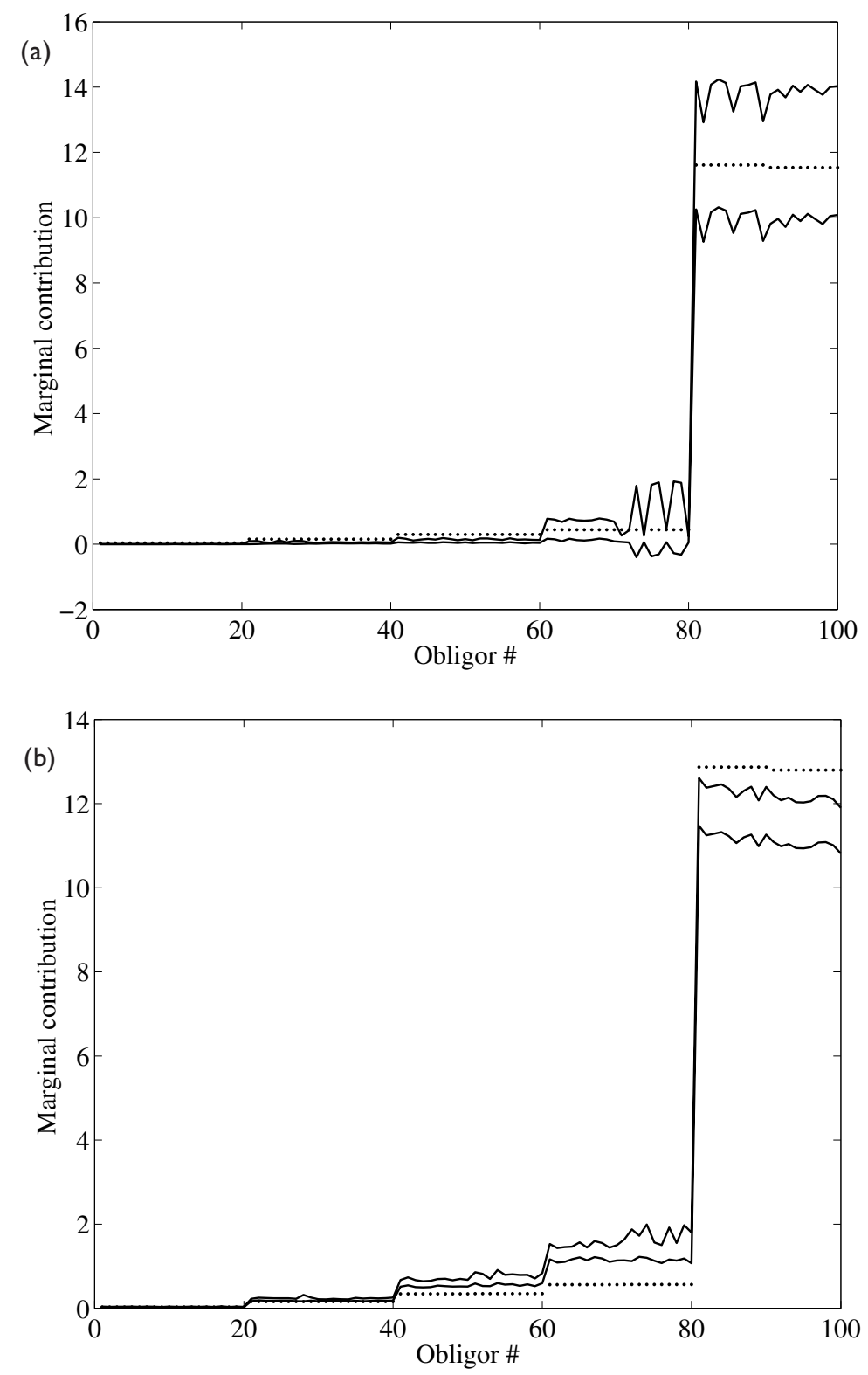
ALGORITHM 5 Approximate VAR contributions.

1. Find $\mu_{*}$ by solving (16); set $z=\mu_{*}$.

2. Calculate $p_{k}(z)$ as in (2), $k=1, \ldots, m$.

3. Solve for $\theta_{x}(z)$ in (11) and calculate $p_{k}\left(\theta_{x}(z), z\right), k=1, \ldots, m$.

4. Return $p_{k}\left(\theta_{x}(Z), Z\right) c_{k}, k=1, \ldots, m$.

ALGORITHM 6 Approximate shortfall contributions.

1. Find $\mu_{*}$ by solving (16) at $x+\sum_{k} p_{k} c_{k}$; set $z=\mu_{*}$.

2. Calculate $p_{k}(z)$ as in $(2), k=1, \ldots, m$.

3. Solve for $\theta_{x}(z)$ in (11) and calculate $p_{k}\left(\theta_{x}^{+}(z), z\right), k=1, \ldots, m$.

4. Return $p_{k}\left(\theta_{x}^{+}(Z), Z\right) c_{k}, k=1, \ldots, m$.

We then approximate expected shortfall contributions as VAR contributions at this shifted threshold $x$.

In Algorithm 6, we evaluate the twisted default probabilities at $\theta_{x}^{+}(z)$ to be consistent with Algorithm 4. However, it is not hard to see that $\theta_{x}(z)$ is positive at $z=\mu_{*}$ (as reflected in the fact that $F_{x}\left(\mu_{*}\right)<0$ in Figure 1), so in this case $\theta_{x}=\theta_{x}^{+}$.

We illustrate these approximations through examples. For Example 1, the approximations of this section coincide with those of Section 8 because there is no dependence on Z; thus, Figure 2 applies to Algorithms 3 and 4. Results for Examples 2-4 are shown in Figures 11-13. The approximations are very good for the VAR contributions, particularly keeping in mind that they are extremely fast.

The approximations are less accurate for expected shortfall contributions (the bottom panel in each figure) than for VAR contributions. This is at least in part due to the fact that we do not know the conditional mean $\mathrm{E}[L \mid L \geq x]$, to which the expected shortfall contributions should sum.

The improvement in the approximation that could result from a better estimate of the conditional mean is illustrated for Example 3 in Figure 14. In this example, we have $x=500$ and $\mathrm{E}[L]=50.5$, so Algorithm 6 evaluates approximate shortfall contributions at 550.5. Using simulation, we estimate that $\mathrm{E}[L \mid L \geq 500]$ is about 713. Evaluating the approximation at this threshold produces the dotted line in the figure, which is nearly indistinguishable from the Monte Carlo results. 
FIGURE II The dashed line shows the approximation. IS confidence intervals (solid lines) are included for reference. The graphs show: (a) VAR contributions; and (b) expected shortfall contributions in Example 2.
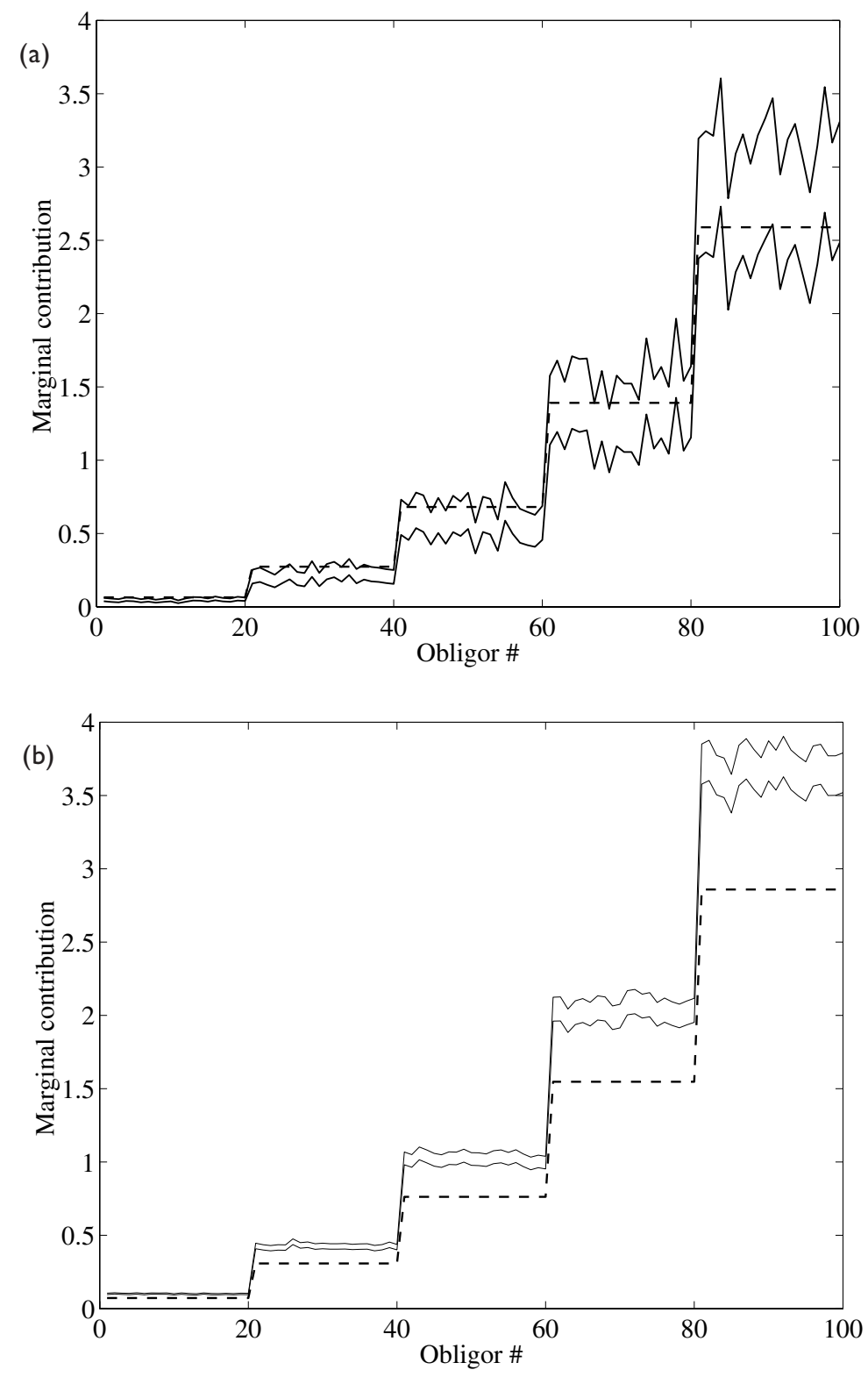
FIGURE 12 The dashed line shows the approximation. IS confidence intervals (solid lines) are included for reference. The graphs show: (a) VAR contributions; and (b) expected shortfall contributions in Example 3.
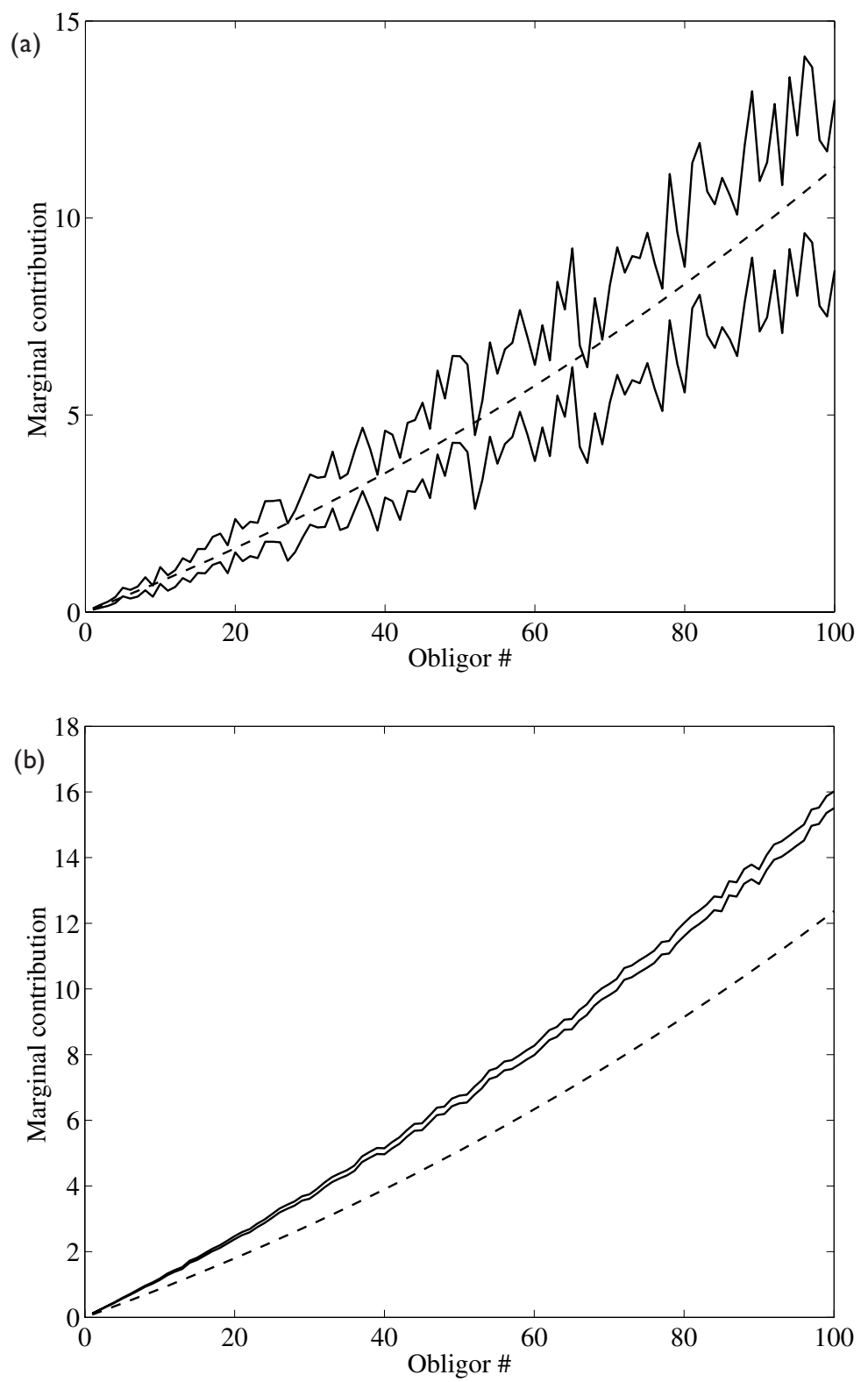
FIGURE 13 The dashed line shows the approximation. IS confidence intervals (solid lines) are included for reference. The graphs show: (a) VAR contributions; and (b) expected shortfall contributions in Example 4.
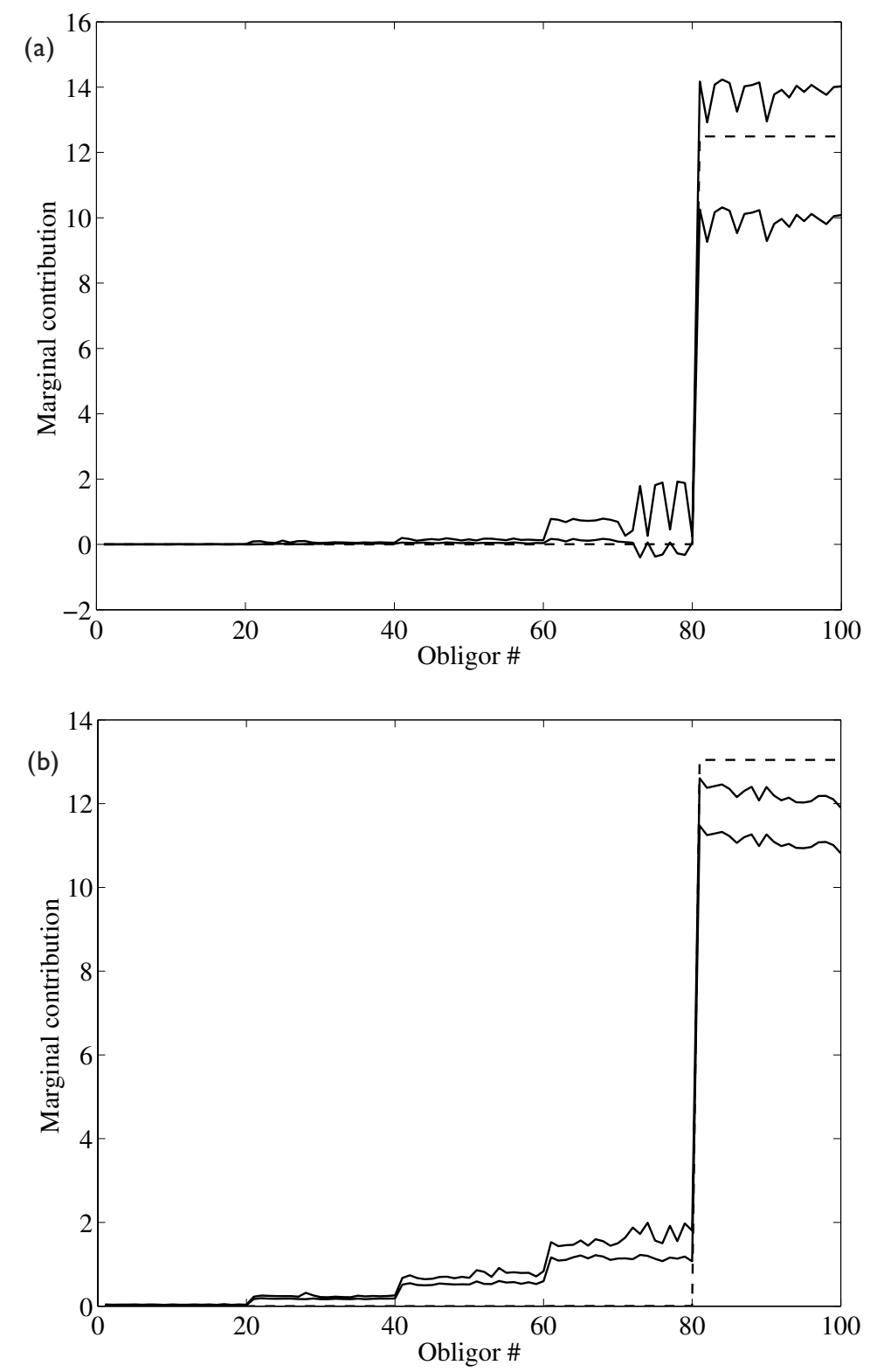
FIGURE I4 The dotted line shows approximate shortfall contributions for Example 3 calculated using $x=713$, the estimated value of $\mathrm{E}[L \mid L \geq 500]$. The dashed line shows the approximation at $x=550.5$. The solid lines are IS confidence intervals.

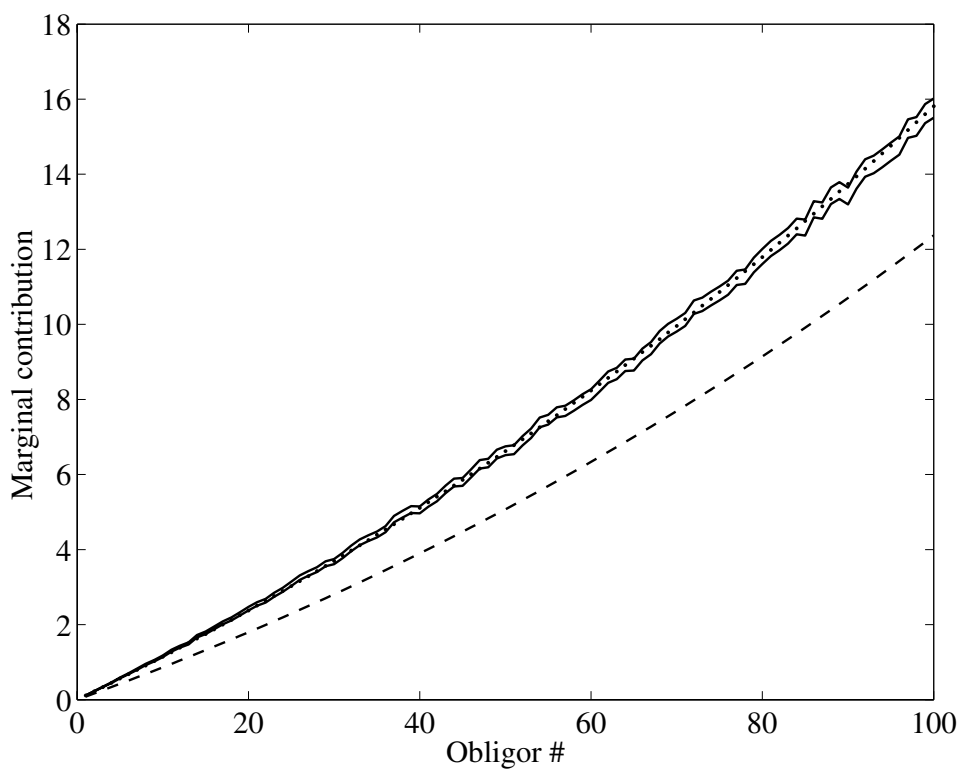

\section{Summary}

We have developed IS methods and approximations for calculating the marginal risk contributions used to allocated portfolio credit risk to individual obligors. We have developed these methods for VAR and expected shortfall in the setting of the Gaussian copula model of portfolio credit risk. We summarize the application of these methods as follows.

- The importance sampling methods are very effective in improving precision in estimating marginal risk contributions. They are particularly effective at high-loss quantiles.

- Combining IS with an asymptotic approximation to conditional default probabilities leads to smoother estimates of the marginal risk contributions. These are quite effective both for VAR and shortfall contributions.

- Similar ideas can be used to approximate marginal risk contributions without Monte Carlo. These approximations are very fast and quite accurate for marginal VAR contributions. They are somewhat less accurate for marginal shortfall contributions.

Both the Monte Carlo methods and the approximations developed here rely on identifying a single "most important" factor outcome. Glasserman et al (2005) 
show that in multi-factor models it is sometimes necessary to consider several "most important" factor outcomes to be used as mean shifts for importance sampling. Although the work in Glasserman et al (2005) applies only to estimating loss probabilities, we expect that the same techniques - based on mixing multiple mean shifts - can be applied to the problem of marginal risk contributions studied here.

\section{REFERENCES}

Artzner, P., Delbaen, F., Eber, J.-M., and Heath, D. (1999). Coherent measures of risk. Mathematical Finance 9, 203-228.

Basel Committee on Bank Supervision (2003). The New Basel Capital Accord, Third Consultative Document, www.bis.org.

Cover, T. M., and Thomas, J. (1991). Elements of Information Theory. Wiley, New York.

Denault, M. (2001). Coherent allocation of risk capital. Journal of Risk 4, 1-34.

Garman, M. (1999). Taking VAR to pieces. Hedging with Trees. Risk Publications, London.

Glasserman, P. (2004). Tail approximations for portfolio credit risk. Journal of Derivatives 12(2), 24-42.

Glasserman, P., Kang, W., and Shahabuddin, P. (2005). Fast simulation of multifactor portfolio credit risk. Working Paper, Columbia University.

Glasserman, P., and Li, J. (2005). Importance sampling for portfolio credit risk. Management Science 51, 1643-1656.

Gourieroux, C., Laurent, J. P., and Scaillet, O. (2000). Sensitivity analysis of values at risk. Journal of Empirical Finance 7, 225-245.

Gupton, G., Finger, C., and Bhatia, M. (1997). CreditMetrics Technical Document, J.P. Morgan $\&$ Co., New York.

Kalkbrener, M. (2003). An axiomatic approach to capital allocation. Mathematical Finance 15, 425-437.

Kalkbrener, M., Lotter, H., and Overbeck, L. (2004). Sensible and efficient capital allocation for credit portfolios. Risk 17, S19-S24.

Kurth, A., and Tasche, D. (2003). Contributions to credit risk. Risk (March), 84-88.

Li, D. (2000). On default correlation: a copula function approach. Journal of Fixed Income $\mathbf{9}$, $43-54$.

Li, J. (2004). Importance sampling for portfolio credit risk. Doctoral Dissertation, Columbia Business School, New York.

Litterman, R. (1999). Hot spots and hedges. Hedging with Trees. Risk Publications, London.

Merton, R. C. (1974). On the pricing of corporate debt: the risk structure of interest rates. Journal of Finance 29, 449-470.

Morokoff, W. J. (2004). An importance sampling method for portfolios of credit risky assets. Proceedings of the Winter Simulation Conference. IEEE Press, Piscataway, New Jersey, pp. 1668-1676. 
Serfling, R. J. (1980). Approximation Theorems of Mathematical Statistics. Wiley, New York.

Van Campenhout, J. M., and Cover, T. M. (1981). Maximum entropy and conditional probability. IEEE Transactions on Information Theory 27, 483-489.

Zabell, S. L. (1980). Rates of convergence for conditional expectations. Annals of Probability 8, 928-941. 